\title{
Maurer-Cartan Forms and the Structure of Lie Pseudo-Groups
}

Peter J. Olver ${ }^{\dagger}$

School of Mathematics

University of Minnesota

Minneapolis, MN 55455

olver@umn.edu

http://www . math. umn. edu/ olver
Juha Pohjanpelto

Department of Mathematics

Oregon State University

Corvallis, OR 97331

juha@math .oregonstate.edu

http: //www. oregonstate.edu/ pohjanpp

Sur la théorie, si importante sans doute, mais pour nous si obscure, des «groupes de Lie infinis》, nous ne savons rien que ce qui se trouve dans les mémoires de Cartan, premiere exploration à travers une jungle presque impénétrable; mais cell-ci menace de se refermer sur les sentiers déjà tracés, si l'on ne procède bientôt à un indispensable travail de défrichement.

- André Weil, [76]

\section{Abstract.}

This paper begins a series devoted to developing a general and practical theory of moving frames for infinite-dimensional Lie pseudo-groups. In this first, preparatory part, we present a new, direct approach to the construction of invariant Maurer-Cartan forms and the Cartan structure equations for a pseudo-group. Our approach is completely explicit and avoids reliance on the theory of exterior differential systems and prolongation.

The second paper [60] will apply these constructions in order to develop the moving frame algorithm for the action of the pseudo-group on submanifolds. The third paper [61] will apply Gröbner basis methods to prove a fundamental theorem on the freeness of pseudo-group actions on jet bundles, and a constructive version of the finiteness theorem of Tresse and Kumpera for generating systems of differential invariants and also their syzygies. Applications of the moving frame method include practical algorithms for constructing complete systems of differential invariants and invariant differential forms, classifying their syzygies and recurrence relations, analyzing invariant variational principles, and solving equivalence and symmetry problems arising in geometry and physics.

$\dagger$ Supported in part by NSF Grant DMS 11-08894.

January 23, 2007 


\section{Introduction.}

Lie pseudo-groups are the infinite-dimensional counterparts of a local Lie groups of transformations. In Lie's day, abstract Lie groups were as yet unknown, and, as a result, no significant distinction was drawn between finite-dimensional and infinite-dimensional theory. However, since then the two subjects have traveled along radically different paths. The finite-dimensional theory has been rigorously formalized, and is a well accepted and widely used mathematical tool. In contrast, the theory of infinite-dimensional pseudo-groups remains surprisingly primitive in its current overall state of development. Since there is still no generally accepted abstract object to play the role of the infinitedimensional Lie group, Lie pseudo-groups only arise through their concrete action on a space. This makes the classification and analytical foundations of the subject problematic, particularly in the intransitive situation. We refer the reader to the original papers of Lie, Medolaghi, and Vessiot, $[\mathbf{4 3}, \mathbf{4 4}, \mathbf{5 1}, \mathbf{7 4}]$, for the classical theory of pseudo-groups, to Cartan, $[\mathbf{1 3}, \mathbf{1 4}]$, for their reformulation in terms of exterior differential systems, and $[21,22,33,34,35,41,42,46,62,63,67,68,70,72]$ for a variety of modern approaches. Lie pseudo-groups appear in many fundamental physical and geometrical contexts, including gauge symmetries, [6], Hamiltonian mechanics and symplectic and Poisson geometry, [54], conformal geometry of surfaces and conformal field theory, $[\mathbf{2 0}, \mathbf{2 3}]$, the geometry of real hypersurfaces, [17], symmetry groups of both linear and nonlinear partial differential equations, such as the Navier-Stokes and Kadomtsev-Petviashvili (KP) equations appearing in fluid and plasma mechanics, $[\mathbf{5}, \mathbf{1 1}, \mathbf{1 9}, \mathbf{5 4}]$, and geometric numerical integration, [50]. Pseudogroups also appear as foliation-preserving groups of transformations, with the associated characteristic classes defined by certain invariant forms, cf. [26].

The main goal of this series of papers is to develop a moving frame theory for Lie pseudo-groups. The motivation behind moving frames or "repères mobiles", as developed by Cartan, [12], is to solve the equivalence problem for submanifolds under the action of a transformation group; see also $[\mathbf{2 8}, \mathbf{2 7}, \mathbf{3 2}]$. In a series of recent collaborative papers, starting with $[\mathbf{2 4}, \mathbf{2 5}]$, the first author has successfully reformulated the classical theory in a completely general, algorithmic, equivariant framework that can be readily applied to arbitrary finite-dimensional Lie group actions. Applications have included complete classifications of differential invariants and their syzygies, equivalence and symmetry properties of submanifolds, rigidity theorems, invariant signatures in computer vision, $[\mathbf{3}, \mathbf{7}, \mathbf{9}, \mathbf{5 7}]$, joint invariants and joint differential invariants, $[\mathbf{8}, \mathbf{5 7}]$, invariant numerical algorithms, $[\mathbf{5 7}, \mathbf{5 8}]$, classical invariant theory, $[\mathbf{4}, \mathbf{5 6}]$, Poisson geometry and solitons, $[\mathbf{4 9}]$, and the calculus of variations, $[\mathbf{3 7}, \mathbf{3 8}]$. New applications of these methods to computation of symmetry groups and classification of partial differential equations can be found in $[\mathbf{4 8}, \mathbf{5 2}]$.

In $[\mathbf{2 4}]$, the preliminary outlines of a comparable theory for infinite-dimensional pseudo-groups was illustrated by a few selected examples. In this series of papers, we shall realize the goal of a complete, rigorous moving frame theory for a wide class of pseudo-group actions. The moving frame theory will allow us to systematically extend all of the cited applications to Lie pseudo-groups, as well as providing new methods and new insights into the foundations of the subject.

Throughout, $M$ will be a smooth manifold, and $\mathcal{G}$ a Lie pseudo-group acting thereon. The action of $\mathcal{G}$ on the submanifolds $S \subset M$ becomes a tale of two (infinite) jet bundles. 
On the one hand, the space of jets of pseudo-group transformations forms a subbundle $\mathcal{G}^{(\infty)} \subset \mathcal{D}^{(\infty)}$ of the bundle $\mathcal{D}^{(\infty)}=\mathcal{D}^{(\infty)}(M) \subset \mathrm{J}^{\infty}(M, M)$ of infinite jets (Taylor series) of local diffeomorphisms $\varphi: M \rightarrow M$. On the other hand, the action of the pseudo-group diffeomorphisms on $p$-dimensional submanifolds $S \subset M$ induces the prolonged pseudogroup action on the corresponding submanifold jet bundle $\mathrm{J}^{\infty}(M, p)$. The ultimate goal of a classical moving frame theory is to analyze the geometry of the prolonged pseudo-group action on $\mathrm{J}^{\infty}(M, p)$.

Our constructions will be based on an amalgamation of two powerful, general theories: groupoids and the variational bicomplex. As first pointed out by Ehresmann, $[\mathbf{2 1}]$, the diffeomorphism jet bundle $\mathcal{D}^{(\infty)}$ carries the structure of a groupoid, $[\mathbf{2 2}, \mathbf{4 7}]$, and $\mathcal{G}^{(\infty)}$ should be viewed as a sub-groupoid thereof. The groupoid category encodes the fact that diffeomorphism jets can only be composed when the target of the first matches the source of the second. Thus, we shall be required to adapt the Lie group based moving frame constructions arising in the finite-dimensional theory into a groupoid version that applies to more general pseudo-groups.

Following $[\mathbf{3 7}, \mathbf{3 8}]$, we shall develop the theory in the context of the variational bicomplex over each (infinite) jet bundle, $[\mathbf{1}, \mathbf{2}, \mathbf{3 8}, \mathbf{7 3}, \mathbf{7 5}]$. The bicomplex construction ${ }^{\dagger}$ relies on a choice of local bundle structure on $M$. The interactions between the two bicomplexes associated with $\mathrm{J}^{\infty}(M, p)$ and $\mathrm{J}^{\infty}(M, M)$ provide the key to understanding the moving frame constructions. In particular, the invariant contact forms on $\mathcal{D}^{(\infty)} \subset \mathrm{J}^{\infty}(M, M)$ will play the role of Maurer-Cartan forms for the diffeomorphism pseudo-group. This identification enables us to formulate explicitly the complete system of structure equations in power series form. Restricting the diffeomorphism-invariant forms to the pseudo-group subbundle $\mathcal{G}^{(\infty)} \subset \mathcal{D}^{(\infty)}$ yields a complete system of Maurer-Cartan forms for the pseudogroup. The remarkable fact is that the Maurer-Cartan forms satisfy an "invariantized" version of the linear infinitesimal determining equations for the pseudo-group, and, as a result, we can immediately produce an explicit form of the pseudo-group structure equations. Our direct algorithm effectively bypasses the more intricate recursive prolongation procedure of Cartan, $[\mathbf{1 3}, \mathbf{1 4}, \mathbf{3 4}, \mathbf{4 5}, \mathbf{4 6}]$. Application of these results to the design of a practical algorithm for directly determining the structure of symmetry (pseudo-)groups of partial differential equations can be found in [15].

The moving frame theory for pseudo-groups acting on submanifold jet bundles will be developed in second paper in this series, [60], which will appear elsewhere. As in the finite-dimensional theory, $[\mathbf{2 5}]$, a moving frame for a pseudo-group action relies on the choice of a cross-section to the pseudo-group orbits. Once the moving frame is determined via normalization of the pseudo-group parameters, the resulting invariantization process leads to a complete system of differential invariants, invariant differential forms, and so on. The all-important recurrence formulae, leading to the determination of a complete,

$\dagger$ An intrinsic formulation requires the more abstract language of spectral sequences, based on the contact filtration of the cotangent bundle, [75]. However, since we shall always deal with local coordinate formulae, we have chosen to keep our constructions within the more down-toearth bicomplex formulation. We refer the reader to Itskov's thesis, $[\mathbf{2 9}, \mathbf{3 0}]$, for the more abstract $\mathcal{C}$-spectral sequence approach. 
finite system of fundamental differential invariants and their syzygies or differential relations, will rely on the Maurer-Cartan form constructions developed here. The fact that we must eventually deal with two distinct jet bundles and their associated bicomplexes complicates the formalism, and establishing a consistent and self-explanatory notation and terminology is half the battle. This is particularly important once we need to consider differential forms, since there are two different systems of contact forms, horizontal forms, total differentiations, etc.

More substantial applications of these results and algorithms are now beginning to appear. In $[\mathbf{1 5}, \mathbf{1 6}]$, our methods are used to analyze symmetry groups of integrable partial differential equations, specifically the Korteweg-deVries (KdV) and KadomtsevPetviashvili (KP) equations, while [5] develops applications to the symmetry groups of partial differential equations arising in fluid mechanics and climatology, modeling shallow water and semi-geostrophic flows. Complete theoretical justifications of our methods will be deferred until the third paper, [61], which relies on new methods from the modern theory of Gröbner bases, [18]. In particular, a new, constructive version of the TresseKumpera finiteness theorem for differential invariants will be established therein.

\section{Pseudo-Groups.}

Let $M$ be a smooth $m$-dimensional manifold, and let $\mathcal{D}=\mathcal{D}(M)$ be the pseudo-group of all local ${ }^{\dagger}$ diffeomorphisms $\varphi: M \rightarrow M$. For each $n \geq 0$, let $\mathcal{D}^{(n)}=\mathcal{D}^{(n)}(M) \subset \mathrm{J}^{n}(M, M)$ denote the bundle of their $n^{\text {th }}$ order jets. In particular $\mathcal{D}^{(0)}=M \times M$. The natural projections are written $\pi_{k}^{n}: \mathcal{D}^{(n)} \rightarrow \mathcal{D}^{(k)}$, and we let $\mathcal{D}^{(\infty)}$ be the inverse limit — the bundle of infinite jets or Taylor series of local diffeomorphisms. Each bundle $\mathcal{D}^{(n)}, 0 \leq n \leq \infty$, carries the structure of a groupoid, $[\mathbf{2 1}, \mathbf{2 2}, \mathbf{4 7}]$. The source map $\boldsymbol{\sigma}^{(n)}\left(\left.\mathrm{j}_{n} \varphi\right|_{z}\right)=z$ and target $\operatorname{map} \boldsymbol{\tau}^{(n)}\left(\left.\mathrm{j}_{n} \varphi\right|_{z}\right)=\varphi(z)=Z$ induce the double fibration

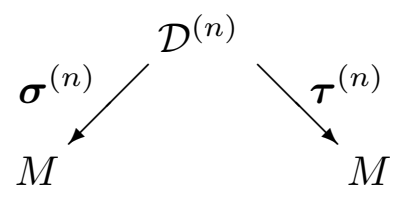

Following Cartan, $[\mathbf{1 3}, \mathbf{1 4}]$, we will consistently use lower case letters, $z, x, u, \ldots$ for the source coordinates and the corresponding upper case letter $Z, X, U, \ldots$ for the target coordinates of our diffeomorphisms $Z=\varphi(z)$.

The groupoid multiplication follows from the composition of local diffeomorphisms. Thus, given $g^{(n)}=\left.\mathrm{j}_{n} \varphi\right|_{z}, h^{(n)}=\left.\mathrm{j}_{n} \psi\right|_{Z}$, with $Z=\boldsymbol{\tau}^{(n)}\left(\left.\mathrm{j}_{n} \varphi\right|_{z}\right)=\boldsymbol{\sigma}^{(n)}\left(\left.\mathrm{j}_{n} \psi\right|_{Z}\right)$, we set $h^{(n)} \cdot g^{(n)}=\left.\mathrm{j}_{n}(\psi \circ \varphi)\right|_{z}$. Let $\mathbb{1}^{(n)}$ denote the identity section of $\boldsymbol{\sigma}^{(n)}: \mathcal{D}^{(n)} \rightarrow M$, whose value at a point $z \in M$ is the $n$-jet $\mathbb{1}_{z}^{(n)}$ of the identity diffeomorphism. Local diffeomorphisms $\psi \in \mathcal{D}$ can act on $\mathcal{D}^{(n)}$ by either left or right multiplication:

$$
\mathrm{L}_{\psi}\left(\left.\mathrm{j}_{n} \varphi\right|_{z}\right)=\left.\mathrm{j}_{n}(\psi \circ \varphi)\right|_{z}, \quad \mathrm{R}_{\psi}\left(\left.\mathrm{j}_{n} \varphi\right|_{z}\right)=\left.\mathrm{j}_{n}\left(\varphi \circ \psi^{-1}\right)\right|_{\psi(z)} .
$$

$\dagger$ Our notational conventions allow the domain of definition of a map $\varphi: M \rightarrow N$ to be a proper open subset: $\operatorname{dom} \varphi \subset M$. Also, when we write $\varphi(z)$ we implicitly assume $z \in \operatorname{dom} \varphi$. 
Given $z \in M$, let $\left.\mathcal{D}^{(n)}\right|_{z}=\left(\boldsymbol{\sigma}^{(n)}\right)^{-1}\{z\}$ denote the source jet fiber. The isotropy sub-group $\mathcal{D}_{z}=\{\varphi \in \mathcal{D} \mid \varphi(z)=z\}$ consists of all local diffeomorphisms that fix $z$. The $n^{\text {th }}$ order frame bundle of $M,[\mathbf{3 6}], \mathcal{F}^{(n)}=\left\{g^{(n)} \in \mathcal{D}^{(n)} \mid \boldsymbol{\sigma}^{(n)}\left(g^{(n)}\right)=\boldsymbol{\tau}^{(n)}\left(g^{(n)}\right)\right\}$ can be identified as the subbundle whose fibers $\left.\mathcal{F}^{(n)}\right|_{z}=\left.\mathcal{D}_{z}^{(n)} \subset \mathcal{D}^{(n)}\right|_{z}$ consist of jets $g^{(n)}=\left.\mathrm{j}_{n} \varphi\right|_{z}$ of isotropy maps $\varphi \in \mathcal{D}_{z}$. For $n<\infty, \mathcal{F}^{(n)}$ forms a principal bundle over $M$ whose structure group is a finite-dimensional Lie group, namely the $n^{\text {th }}$ order prolonged linear group

$$
\mathrm{GL}^{(n)}(m)=\left\{\left.\mathrm{j}_{n} \varphi\right|_{0} \mid \varphi \in \mathcal{D}\left(\mathbb{R}^{m}\right), \varphi(0)=0\right\}
$$

prescribed by $n$-jets of local diffeomorphisms $\varphi: \mathbb{R}^{m} \rightarrow \mathbb{R}^{m}$ that fix the origin, [36; p. 139], $[\mathbf{5 3}, \mathbf{5 9}]$. The group multiplication is induced by composition of diffeomorphisms.

Given local coordinates $(z, Z)=\left(z^{1}, \ldots, z^{m}, Z^{1}, \ldots, Z^{m}\right)$ on an open subset of $M \times M$, the induced local Taylor coordinates of $g^{(n)}=\left.\mathrm{j}_{n} \varphi\right|_{z} \in \mathcal{D}^{(n)}$ are denoted $\left(z, Z^{(n)}\right)$, where the components $Z_{J}^{a}$ of $Z^{(n)}$, for $a=1, \ldots, m$, \#J $\leq n$, represent the partial derivatives $\partial^{J} \varphi^{a} / \partial z^{J}$ of the components $\varphi^{a}\left(z^{1}, \ldots, z^{m}\right)$ of the map $\varphi$ at the source point $z=$ $\boldsymbol{\sigma}^{(n)}\left(g^{(n)}\right)$. We adopt a symmetric multi-index notation for derivatives, so $J=\left(j_{1}, \ldots, j_{k}\right)$ denotes an unordered $k=\# J$-tuple with entries $1 \leq j_{\nu} \leq m=\operatorname{dim} M$. Note that

$$
\operatorname{dim} \mathcal{F}^{(n)}=m\left(\begin{array}{c}
m+n \\
m
\end{array}\right), \quad \operatorname{dim} \mathcal{D}^{(n)}=m\left[1+\left(\begin{array}{c}
m+n \\
m
\end{array}\right)\right] .
$$

Example 2.1. Consider the case $M=\mathbb{R}$ with coordinate $x$. Local coordinates of $g^{(n)}=\left.\mathrm{j}_{n} \varphi\right|_{x} \in \mathcal{D}^{(n)}(\mathbb{R})$ are denoted

$$
\left(x, X, X_{x}, X_{x x}, \ldots, X_{n}\right),
$$

where $X_{k}$ corresponds to the $k^{\text {th }}$ derivative of the diffeomorphism $X=\varphi(x)$ at the source point $x$. An infinite jet $g^{(\infty)}=\left(x, X^{(\infty)}\right) \in \mathcal{D}^{(\infty)}$ corresponds to the Taylor series

$$
X \llbracket h \rrbracket=X+X_{x} h+\frac{1}{2} X_{x x} h^{2}+\cdots
$$

of a diffeomorphism $X=\varphi(x+h)$ at the source point $x$. Multiplication of diffeomorphism jets corresponds to composition (and, in the finite order case, truncation) of power series ${ }^{\dagger}$,

$$
\left(X, \mathbf{X}, \mathbf{X}_{X}, \mathbf{X}_{X X}, \ldots\right) \cdot\left(x, X, X_{x}, X_{x x}, \ldots\right)=\left(x, \mathbf{X}, \mathbf{X}_{X} X_{x}, \mathbf{X}_{X} X_{x x}+\mathbf{X}_{X X} X_{x}^{2}, \ldots\right) .
$$

The higher-order terms can be expressed in terms of Bell polynomials via the general Fàadi-Bruno formula, $[\mathbf{5 9}, \mathbf{6 5}]$. Note that the groupoid multiplication (2.6) only makes sense when the source coordinate of the first jet matches the target coordinate of the second.

Roughly, a Lie pseudo-group $\mathcal{G} \subset \mathcal{D}$ is a sub-pseudo-group defined by an involutive system of partial differential equations, $[\mathbf{2 1}, \mathbf{2 2}, \mathbf{3 9}, \mathbf{6 2}, \mathbf{6 3}, \mathbf{6 4}]$. For each $0 \leq n \leq \infty$, we let $\mathcal{G}^{(n)} \subset \mathcal{D}^{(n)}$ denote the sub-groupoid consisting of all $n$-jets of pseudo-group diffeomorphisms. For $n<\infty$, we can regard the subbundle $\mathcal{G}^{(n)}$ as representing a system of $n^{\text {th }}$

$\dagger$ The reader may recoil at this XXX rated formula, but if we fail to take a consistent and systematic stance on notation at the outset, we are doomed to complete confusion before the end. 
order partial differential equations, known as the determining equations of order $n$ for the pseudo-group. Given $z \in M$, let $\mathcal{G}_{z}=\mathcal{G} \cap \mathcal{D}_{z}$ denote the isotropy subgroup consisting of those pseudo-group transformations that fix the point $z \in M$, and $\mathcal{G}_{z}^{(n)}=\left.\mathcal{G}^{(n)} \cap \mathcal{F}^{(n)}\right|_{z}$ the subgroup of isotropy jets at $z$.

There is some disagreement over technical hypotheses in the definition of a Lie pseudogroup in the literature. Our version is stated as follows:

Definition 2.2. A sub-pseudo-group $\mathcal{G} \subset \mathcal{D}$ will be called a Lie pseudo-group if there exists $n^{\star} \geq 1$ such that the following assumptions are satisfied for all finite $n \geq n^{\star}$ :

(a) $\mathcal{G}^{(n)} \subset \mathcal{D}^{(n)}$ forms a smooth, embedded subbundle,

(b) $\pi_{n}^{n+1}: \mathcal{G}^{(n+1)} \rightarrow \mathcal{G}^{(n)}$ is a bundle map,

(c) every smooth local solution $Z=\varphi(z)$ to the determining system $\mathcal{G}^{(n)}$ belongs to $\mathcal{G}$,

(d) $\mathcal{G}^{(n)}=\operatorname{pr}^{\left(n-n^{\star}\right)} \mathcal{G}^{\left(n^{\star}\right)}$ is obtained by prolongation.

The conditions $(a-d)$ codify the formal integrability and local solvability requirements placed on the determining equations for the pseudo-group. In this paper, we shall not require the more technical condition of involutivity, as based on Janet-Riquier theory, $[\mathbf{3 1}, \mathbf{6 6}]$, Spencer cohomology, $[\mathbf{6 2}, \mathbf{6 3}]$, or involutive divisions, [10].

The minimal value of $n^{\star}$ is called the order of the pseudo-group, as it specifies the order of the determining system of partial differential equations that characterizes the pseudo-group transformations. We note that hypothesis (a) implies that the isotropy jets $\left.\mathcal{G}_{z}^{(n)} \subset \mathcal{F}^{(n)}\right|_{z}$ form a finite-dimensional Lie group for each $z \in M$.

Remark: The modern approach to pseudo-groups, $[\mathbf{2 1}, \mathbf{2 2}, \mathbf{3 9}, \mathbf{4 1}, \mathbf{4 2}, \mathbf{6 2}, \mathbf{7 0}]$, has, by and large, been founded upon the theory of $G$-structures, [71]. Pseudo-groups that arise in this manner are necessarily of constant type, meaning that the isotropy jet groups $\mathcal{G}_{z}^{(n)}$ are conjugate subgroups of $\mathrm{GL}^{(n)}(m)$. In his definition of a continuous pseudo-group, Kuranishi, [41], makes the less restrictive requirement that $\operatorname{dim} \mathcal{G}_{z}^{(n)}$ is constant on $M$. However, the Cartan construction of essential invariants, $[\mathbf{1 3}, \mathbf{7 2}]$, demonstrates that such an assumption is overly restrictive in the intransitive case. A key feature of our approach is that this constant dimensionality condition is not required, and, in fact, appears to play no significant role in the general theory!

Remark: In his thesis, [30], V. Itskov proves that, under suitable hypotheses, any non-Lie pseudo-group has the same differential invariants as a certain larger Lie pseudogroup, known as its completion; see also Johnson, [33; p. 317]. Thus, we are not sacrificing any significant generality by restricting our attention to Lie pseudo-groups in our study.

In local coordinates, the order $n$ determining equations defining the pseudo-group subbundle $\mathcal{G}^{(n)}$ take the form of a system of nonlinear partial differential equations

$$
F^{(n)}\left(z, Z^{(n)}\right)=0
$$

whose local solutions $Z=\varphi(z)$ are, for any $n \geq n^{\star}$, the pseudo-group transformations. The prolonged system $\mathrm{pr}^{(k)} \mathcal{G}^{(n)}$ is obtained by repeatedly applying the total differential 
operators $^{\dagger}$

$$
\mathbb{D}_{z^{i}}=\frac{\partial}{\partial z^{i}}+\sum_{a=1}^{m} \sum_{\# J \geq 0} Z_{J, i}^{a} \frac{\partial}{\partial Z_{J}^{a}}, \quad i=1, \ldots, m
$$

The integrability condition $(d)$ of the definition requires that, for all $n \geq n^{\star}$, the system $\operatorname{pr}^{(k)} \mathcal{G}^{(n)}$ obtained by $k$-fold differentiation is the same as $\mathcal{G}^{(n+k)}$. We remark that the system of partial differential equations (2.7) must satisfy the constraints that it be a nonlinear Lie equation, [40], ensuring that the composition of any two solutions must, where defined, also be a solution.

To illustrate our constructions, we will primarily focus on two elementary, but nevertheless interesting examples of pseudo-groups. Our methods, of course, have far wider applicability, and these examples have merely been chosen for their inherent simplicity.

Example 2.3. Let $M=\{(x, u) \mid u \neq 0\} \subset \mathbb{R}^{2}$. Consider the transitive Lie pseudogroup $\mathcal{G} \subset \mathcal{D}(M)$ consisting of (local) diffeomorphisms of the form

$$
X=f(x), \quad U=\frac{u}{f^{\prime}(x)},
$$

where $f(x) \in \mathcal{D}(\mathbb{R})$ is an arbitrary local diffeomorphism. This example was introduced by Lie, [43; p. 353], in his classification of infinite-dimensional pseudo-groups acting on the plane. The pseudo-group jets are obtained by repeated differentiation; to second order,

$$
\begin{aligned}
& X=f, \quad U=\frac{u}{f_{x}}, \quad X_{x}=f_{x}, \quad X_{u}=0, \quad U_{x}=-\frac{u f_{x x}}{f_{x}^{2}}, \quad U_{u}=\frac{1}{f_{x}}, \quad(2.10) \\
& X_{x x}=f_{x x}, \quad X_{x u}=X_{u u}=0, \quad U_{x x}=-\frac{u f_{x x x}}{f_{x}^{2}}+\frac{2 u f_{x x}^{2}}{f_{x}^{3}}, \quad U_{x u}=-\frac{f_{x x}}{f_{x}^{2}}, \quad U_{u u}=0,
\end{aligned}
$$

where $x, u, X, U, X_{x}, X_{u}, U_{x}, U_{u}, X_{x x}, \ldots$ denote local coordinates on $\mathcal{D}^{(n)}(M)$. In the transformation formulae, the derivatives $f, f_{x}, f_{x x}, \ldots$ represent the coefficients in the Taylor series for the function $f(x+h)$ at the source point, and should be viewed as independent parameters; in particular, when not followed by $(x)$, the symbol $f=f_{0}$ represents the 0 -jet or constant term in the Taylor series. Thus, the $n^{\text {th }}$ order jet groupoid $\mathcal{G}^{(n)} \subset \mathcal{D}^{(n)}$ forms a $(n+4)$-dimensional subbundle parametrized by the source coordinates $x, u$ and the Taylor parameters $f, f_{x}, f_{x x}, \ldots$

The involutive system characterizing $\mathcal{G}^{(n)}$ is obtained by implicitization, and equals the $(n-1)^{\text {st }}$ prolongation of the involutive first order system

$$
X_{x}=\frac{u}{U}, \quad X_{u}=0, \quad U_{u}=\frac{U}{u},
$$

$\dagger$ The notation will serve to distinguish these total derivatives from those on the submanifold jet bundle $\mathrm{J}^{n}(M, p)$ to be used in [60]. A more intrinsic characterization appears in (3.3) below. 
obtained by recursively applying the total derivatives

$$
\begin{aligned}
& \mathbb{D}_{x}=\frac{\partial}{\partial x}+X_{x} \frac{\partial}{\partial X}+U_{x} \frac{\partial}{\partial U}+X_{x x} \frac{\partial}{\partial X_{x}}+X_{x u} \frac{\partial}{\partial X_{u}}+U_{x x} \frac{\partial}{\partial U_{x}}+U_{x u} \frac{\partial}{\partial U_{u}}+\cdots \\
& \mathbb{D}_{u}=\frac{\partial}{\partial u}+X_{u} \frac{\partial}{\partial X}+U_{u} \frac{\partial}{\partial U}+X_{x u} \frac{\partial}{\partial X_{x}}+X_{u u} \frac{\partial}{\partial X_{u}}+U_{x u} \frac{\partial}{\partial U_{x}}+U_{u u} \frac{\partial}{\partial U_{u}}+\cdots
\end{aligned}
$$

For instance, its first prolongation $\operatorname{pr}^{(1)} \mathcal{G}^{(1)}=\mathcal{G}^{(2)}$ is the six-dimensional submanifold of $\mathcal{D}^{(2)}$ defined implicitly by appending the second order conditions

$$
X_{x x}=-\frac{u U_{x}}{U^{2}}, \quad X_{x u}=X_{u u}=0, \quad U_{x u}=\frac{U_{x}}{u}, \quad U_{u u}=0 .
$$

Example 2.4. Our second illustrative example is the Lie pseudo-group $\mathcal{G}$ consisting of local diffeomorphisms of the form

$$
X=f(x), \quad Y=f^{\prime}(x) y+g(x), \quad U=u+\frac{f^{\prime \prime}(x) y+g^{\prime}(x)}{f^{\prime}(x)},
$$

acting on $M \simeq \mathbb{R}^{3}$. Here $f(x) \in \mathcal{D}(\mathbb{R})$ is an arbitrary local diffeomorphism of $\mathbb{R}$, while $g(x) \in \mathrm{C}^{\infty}(\mathbb{R})$ is an arbitrary smooth function of a single variable $x$. This example generalizes one of Medolaghi's pseudo-groups, [51], and illustrates some of the more subtle features in our constructions. It also serves as a simplified version of the symmetry pseudogroup of the KP equation, [15], and captures many of the features of the latter. The prolonged transformations are determined by differentiating the defining equations (2.14) with respect to $x, y, u$, keeping in mind that $f_{y}=f_{u}=g_{y}=g_{u}=0$. Consequently, the subbundle $\mathcal{G}^{(n)} \subset \mathcal{D}^{(n)}$ is parametrized by the Taylor coordinates $x, y, u, f, g, f_{x}, g_{x}$, $f_{x x}, g_{x x}, \ldots, f_{n+2}, g_{n+1}$. However, at order $n$, the parameter $f_{n+2}$ is redundant, since only the particular combination $f_{n+2} y+g_{n+1}$ appears in the formulae. Eliminating the parametric coordinates, we find that for $n \geq 1$, the jet groupoid $\mathcal{G}^{(n)}$ is the $(2 n+7)$ dimensional subbundle of $\mathcal{D}^{(n)}$ defined by prolonging the first order system

$$
X_{y}=X_{u}=0, \quad Y_{y}=X_{x} \neq 0, \quad Y_{u}=0, \quad Y_{x}=(U-u) X_{x}, \quad U_{u}=1,
$$

through successive application of the total derivatives $\mathbb{D}_{x}, \mathbb{D}_{y}, \mathbb{D}_{u}$.

\section{Maurer-Cartan Forms for the Diffeomorphism Groupoid.}

As emphasized in [38], the moving frame calculus for finite-dimensional Lie groups relies in an essential manner on the invariant Maurer-Cartan forms. Thus, the moving frame theory for pseudo-groups requires a corresponding collection of invariant differential forms on the jet groupoids. Our constructions are motivated by the original ideas of Cartan, $[\mathbf{1 3}, \mathbf{1 4}, \mathbf{3 4}, \mathbf{7 2}]$. However, in contrast to Cartan's recursive algorithm based upon successive prolongations of the underlying exterior differential system, we will give a direct, explicit construction of the invariant forms on the infinite diffeomorphism jet bundle $\mathcal{D}^{(\infty)}$.

Since we can identify $\mathcal{D}^{(\infty)} \subset \mathrm{J}^{\infty}(M, M)$ as (an open subset of) a jet bundle over a Cartesian product manifold, there is a natural splitting of the cotangent bundle $T^{*} \mathcal{D}^{(\infty)}$ 
into horizontal and vertical (contact) components, $[\mathbf{1}, \mathbf{5 5}]$. We use ${ }^{\dagger}$

$$
d=d_{M}+d_{G}
$$

to denote the consequent splitting of the differential on $\mathcal{D}^{(\infty)}$. The subscript on the vertical differential $d_{G}$ refers to the groupoid structure of $\mathcal{D}^{(\infty)}$. The following interpretation is fundamental:

The vertical differential $d_{G}$ on $\mathcal{D}^{(\infty)}$ should be viewed as differentiation with respect to the pseudo-group parameters, and hence plays the role of the group differential $d_{G}$ in the finite-dimensional theory, [38]. Moreover, the Maurer-Cartan forms for the diffeomorphism pseudo-group will be concretely realized as the (right-)invariant contact one-forms on $\mathcal{D}^{(\infty)}$.

In terms of local coordinates $g^{(\infty)}=\left(z, Z^{(\infty)}\right)$, the horizontal subbundle of $T^{*} \mathcal{D}^{(\infty)}$ is spanned by the one-forms $d z^{1}=d_{M} z^{1}, \ldots, d z^{m}=d_{M} z^{m}$, while the vertical subbundle is spanned by the basic contact forms

$$
\Upsilon_{J}^{a}=d_{G} Z_{J}^{a}=d Z_{J}^{a}-\sum_{i=1}^{m} Z_{J, i}^{a} d z^{i}, \quad a=1, \ldots, m, \quad \# J \geq 0 .
$$

Given a differential function $F: \mathcal{D}^{(\infty)} \rightarrow \mathbb{R}$, its horizontal and vertical differentials take the form

$$
d_{M} F=\sum_{i=1}^{m} \mathbb{D}_{z^{i}} F d z^{i}, \quad d_{G} F=\sum_{a=1}^{m} \sum_{\# J \geq 0} \frac{\partial F}{\partial Z_{J}^{a}} \Upsilon_{J}^{a}
$$

where $\mathbb{D}_{z^{i}}$ are the total differentiation operators (2.8). In particular,

$$
d_{M} Z_{J}^{a}=\sum_{i=1}^{m} Z_{J, i}^{a} d z^{i}
$$

Note that the higher-order contact forms (3.2) are obtained by iterated Lie differentiation: $\Upsilon_{J}^{a}=\mathbb{D}_{z}^{J} \Upsilon^{a}$, where $\mathbb{D}_{z}^{J}=\mathbb{D}_{z^{j_{1}}} \cdots \mathbb{D}_{z^{j_{k}}}$ denotes a $k^{\text {th }}$ order total derivative.

Definition 3.1. A differential form $\mu$ on $\mathcal{D}^{(n)}, 0 \leq n \leq \infty$, is right-invariant if it satisfies $\left(\mathrm{R}_{\psi}\right)^{*} \mu=\mu$, where defined, for every local diffeomorphism $\psi \in \mathcal{D}$.

Remark: Morally, right-invariance of differential forms should be with respect to the right groupoid multiplication. This certainly is the case for functions. However, since the domain of a groupoid element is not an open subset of $\mathcal{D}^{(\infty)}$, defining the pull-back groupoid action on differential forms becomes problematic.

Note that the splitting of forms on $\mathcal{D}^{(\infty)}$ into horizontal and contact components is also invariant under the action of $\mathcal{D}$ on $\mathcal{D}^{(n)}$. Hence, if $\mu$ is any right-invariant differential

$\dagger$ We reserve the more traditional notation $d_{H}$ and $d_{V}$ for the splitting of the submanifold jet bundle $\mathrm{J}^{\infty}(M, p)$ to be used in $[\mathbf{6 0}]$. 
form, so are $d_{M} \mu$ and $d_{G} \mu$. Now, the target coordinate functions $Z^{a}: \mathcal{D}^{(0)} \rightarrow \mathbb{R}$ are obviously right-invariant. Therefore, by the preceding remark, their differentials

$$
d Z^{a}=d_{M} Z^{a}+d_{G} Z^{a} \equiv \sigma^{a}+\mu^{a}, \quad a=1, \ldots, m,
$$

split into right-invariant horizontal and contact forms. Thus, the one-forms

$$
\sigma^{a}=d_{M} Z^{a}=\sum_{i=1}^{m} Z_{i}^{a} d z^{i}, \quad a=1, \ldots, m,
$$

form an invariant horizontal coframe, while

$$
\mu^{a}=d_{G} Z^{a}=\Upsilon^{a}=d Z^{a}-\sum_{i=1}^{m} Z_{i}^{a} d z^{i}, \quad a=1, \ldots, m,
$$

are the zero ${ }^{\text {th }}$ order invariant contact forms. The total differential operators $\mathbb{D}_{Z^{1}}, \ldots, \mathbb{D}_{Z^{m}}$ dual to the horizontal forms $\sigma_{1}, \ldots, \sigma_{m}$ are defined by the formula

$$
d_{M} F=\sum_{a=1}^{m} \mathbb{D}_{Z^{a}} F \sigma^{a}
$$

Therefore,

$$
\mathbb{D}_{Z^{a}}=\sum_{i=1}^{m} w_{a}^{i} \mathbb{D}_{z^{i}}, \quad \text { where } \quad\left(w_{a}^{i}\left(z, Z^{(1)}\right)\right)=\left(Z_{i}^{a}\right)^{-1}
$$

denotes the inverse of the $m \times m$ Jacobian matrix $\nabla Z=\left(Z_{i}^{a}\right)$.

Invariance of the forms (3.6) implies that the Lie derivative of a right-invariant differential form with respect to $\mathbb{D}_{Z^{a}}$ will also be right-invariant. Therefore, the higher-order invariant contact forms are

$$
\mu_{J}^{a}=\mathbb{D}_{Z}^{J} \mu^{a}=\mathbb{D}_{Z}^{J} \Upsilon^{a}, \quad \text { where } \quad \mathbb{D}_{Z}^{J}=\mathbb{D}_{Z^{j_{1}}} \cdots \mathbb{D}_{Z^{j_{k}}}, \quad \begin{array}{ll}
a=1, \ldots, m, \\
k=\# J \geq 0 .
\end{array}
$$

The differential operators $\mathbb{D}_{Z^{j}}$ mutually commute, and so the order of differentiation is immaterial. We shall view the right-invariant contact forms $\mu^{(\infty)}=\left(\ldots \mu_{J}^{a} \ldots\right)$ on $\mathcal{D}^{(\infty)}$ as the Maurer-Cartan forms for the diffeomorphism pseudo-group. They will play exactly the same role that the ordinary Maurer-Cartan forms played in the construction of the invariant variational bicomplex in the finite-dimensional Lie group theory, [38]. The complete collection $\sigma, \mu^{(\infty)}$ in (3.6), (3.10) forms a right-invariant coframe on $\mathcal{D}^{(\infty)}$.

Example 3.2. Consider the one-dimensional case $M=\mathbb{R}$. Using the coordinate notation of Example 2.1, the first few contact forms on $\mathcal{D}^{(\infty)}$ are

$$
\Upsilon=d_{G} X=d X-X_{x} d x, \Upsilon_{x}=\mathbb{D}_{x} \Upsilon=d X_{x}-X_{x x} d x, \Upsilon_{x x}=\mathbb{D}_{x}^{2} \Upsilon=d X_{x x}-X_{x x x} d x
$$

where

$$
\mathbb{D}_{x}=\frac{\partial}{\partial x}+X_{x} \frac{\partial}{\partial X}+X_{x x} \frac{\partial}{\partial X_{x}}+X_{x x x} \frac{\partial}{\partial X_{x x}}+\cdots
$$


The basic right-invariant horizontal form is

$$
\sigma=d_{M} X=X_{x} d x, \quad \text { with dual total differentiation } \quad \mathbb{D}_{X}=\frac{1}{X_{x}} \mathbb{D}_{x} .
$$

Our algorithm produces the basic right-invariant Maurer-Cartan forms

$$
\mu=\Upsilon, \quad \mu_{X}=\mathbb{D}_{X} \mu=\frac{\Upsilon_{x}}{X_{x}}, \quad \mu_{X X}=\mathbb{D}_{X}^{2} \mu=\frac{X_{x} \Upsilon_{x x}-X_{x x} \Upsilon_{x}}{X_{x}^{3}}, \quad \ldots
$$

Remark: While the right-invariant forms underlie the moving frame calculus, a similar construction applies to differential forms invariant under the left action of $\mathcal{D}$ on $\mathcal{D}^{(\infty)}$. The source coordinates $z^{i}$ are left-invariant, and hence their differentials

$$
d z^{i}=w_{a}^{i} d Z^{a}-\sum_{a=1}^{m} w_{a}^{i} \Upsilon^{a}, \quad i=1, \ldots, m,
$$

where $w_{a}^{i}$ is defined in (3.9), split into left invariant one-forms. A full system of leftinvariant forms is obtained by further Lie differentiation:

$$
\psi^{i}=d z^{i}, \quad \lambda^{i}=\sum_{a=1}^{m} w_{a}^{i} \Upsilon^{a}, \quad \text { and } \quad \lambda_{J}^{i}=\mathbb{D}_{z}^{J} \lambda^{i} .
$$

In the one-dimensional situation of Example 3.2, the first few left-invariant one-forms are

$$
\begin{gathered}
\psi=d x, \quad \lambda=\frac{\Upsilon}{X_{x}}=\frac{d X-X_{x} d x}{X_{x}}, \\
\lambda_{x}=\frac{X_{x} \Upsilon_{x}-X_{x x} \Upsilon}{X_{x}^{2}}, \quad \lambda_{x x}=\frac{X_{x}^{2} \Upsilon_{x x}-2 X_{x} X_{x x} \Upsilon_{x}-\left(X_{x} X_{x x x}-2 X_{x x}^{2}\right) \Upsilon}{X_{x}^{3}} .
\end{gathered}
$$

\section{The Diffeomorphism Structure Equations.}

The next step in our program is to establish the structure equations for the rightinvariant Maurer-Cartan forms on the diffeomorphism groupoid $\mathcal{D}^{(\infty)}$. Instead of following Cartan's iterative procedure based on successive prolongation of Pfaffian systems, [13], we shall present a direct approach based on a power series analysis.

Given local coordinates $z=\left(z^{1}, \ldots, z^{m}\right)$ on $M$, we use $Z \llbracket h \rrbracket$ to denote the vectorvalued Taylor series, depending on $h=\left(h^{1}, \ldots, h^{m}\right)$, of a diffeomorphism $Z=\varphi(z+h)$ at the source point $z \in M$, with components

$$
Z^{a} \llbracket h \rrbracket=\sum_{\# J \geq 0} \frac{1}{J !} Z_{J}^{a} h^{J}, \quad a=1, \ldots, m .
$$

Similarly, the Taylor series $\mathbf{Z} \llbracket H \rrbracket$ of a diffeomorphism $\mathbf{Z}=\psi(Z+H)$ based at $Z$ has components

$$
\mathbf{Z}^{a} \llbracket H \rrbracket=\sum_{\# J \geq 0} \frac{1}{J !} \mathbf{Z}_{J}^{a} H^{J}, \quad a=1, \ldots, m,
$$


with $H=\left(H^{1}, \ldots, H^{m}\right)$. The groupoid multiplication on $\mathcal{D}^{(\infty)}$ is induced by composition of power series, with $\mathbf{Z} \llbracket Z \llbracket h \rrbracket-Z \llbracket 0 \rrbracket \rrbracket$ being the Taylor series of the composition $\psi \circ \varphi$.

Let $\Upsilon \llbracket h \rrbracket$ denote the column vector of contact form-valued power series with individual components

$$
\Upsilon^{a} \llbracket h \rrbracket=\sum_{\# J \geq 0} \frac{1}{J !} \Upsilon_{J}^{a} h^{J}, \quad a=1, \ldots, m .
$$

Similarly, $\mu \llbracket H \rrbracket$ will denote the right-invariant contact form-valued power series with

$$
\mu^{a} \llbracket H \rrbracket=\sum_{\# J \geq 0} \frac{1}{J !} \mu_{J}^{a} H^{J}, \quad a=1, \ldots, m .
$$

Equations (3.10) imply that

$$
\mu \llbracket H \rrbracket=\Upsilon \llbracket h \rrbracket \quad \text { when } \quad H=Z \llbracket h \rrbracket-Z \llbracket 0 \rrbracket,
$$

which can be used to recover the Maurer-Cartan forms (3.10).

To obtain the structure equations for the diffeomorphism pseudo-group, we apply the exterior derivative $d$ (with respect to the source variables $z$ ) to $\mu \llbracket H \rrbracket$ while keeping $h$ fixed. First note that, from (3.2), (3.4),

$$
d H=d Z \llbracket h \rrbracket-d Z \llbracket 0 \rrbracket=d_{M} Z \llbracket h \rrbracket+d_{G} Z \llbracket h \rrbracket-d Z \llbracket 0 \rrbracket=\nabla_{h} Z \llbracket h \rrbracket d z+\Upsilon \llbracket h \rrbracket-d Z \llbracket 0 \rrbracket .
$$

Here

$$
\nabla_{h} Z \llbracket h \rrbracket=\left(\frac{\partial Z^{a}}{\partial h^{i}} \llbracket h \rrbracket\right)
$$

denotes the $m \times m$ Jacobian matrix power series obtained by differentiating $Z \llbracket h \rrbracket$ with respect to $h=\left(h^{1}, \ldots, h^{m}\right)$, while $d z=\left(d z^{1}, \ldots, d z^{m}\right)^{T}$ is viewed as a column vector of one-forms. Using this notation, the standard structure equations

$$
d \Upsilon_{J}^{a}=-\sum_{i=1}^{m} \Upsilon_{J, i}^{a} \wedge d z^{i}
$$

for the contact forms on $\mathcal{D}^{(\infty)},[\mathbf{5 5}]$, can be written in power series form as

$$
d \Upsilon \llbracket h \rrbracket=-\nabla_{h} \Upsilon \llbracket h \rrbracket \wedge d z .
$$

Therefore, differentiating (4.5) and using the chain rule, we find

$$
d \mu \llbracket H \rrbracket-\nabla_{H} \mu \llbracket H \rrbracket \wedge d H=d(\mu \llbracket H \rrbracket)=d \Upsilon \llbracket h \rrbracket=-\nabla_{h} \Upsilon \llbracket h \rrbracket \wedge d z .
$$

It is important to note that, in the first term $d \mu \llbracket H \rrbracket$ in (4.9), the exterior derivative only affects the invariant forms $\mu$, whereas in the middle term $d(\mu \llbracket H \rrbracket)$ it affects both $\mu$ and $H$. In view of $(4.6)$, we obtain the structure equations in the form

$$
\begin{aligned}
d \mu \llbracket H \rrbracket & =\nabla_{H} \mu \llbracket H \rrbracket \wedge\left(\nabla_{h} Z \llbracket h \rrbracket d z+\Upsilon \llbracket h \rrbracket-d Z \llbracket 0 \rrbracket\right)-\nabla_{h} \Upsilon \llbracket h \rrbracket \wedge d z \\
& =\nabla_{H} \mu \llbracket H \rrbracket \wedge(\mu \llbracket H \rrbracket-d Z \llbracket 0 \rrbracket)
\end{aligned}
$$


where the first and last terms canceled thanks to (4.5) and the chain rule. The constant term in the final power series is

$$
\mu \llbracket 0 \rrbracket-d Z \llbracket 0 \rrbracket=-\sigma,
$$

whose components are the negatives of the invariant horizontal forms $-\sigma^{a}$ defined in (3.6). Therefore, their structure equations are obtained by setting $H=0$ in (4.10):

$$
d \sigma=-d \mu \llbracket 0 \rrbracket=\nabla_{H} \mu \llbracket 0 \rrbracket \wedge \sigma .
$$

Equations (4.10), (4.11) constitute the complete system of structure equations for the diffeomorphism pseudo-group.

Example 4.1. When $M=\mathbb{R}$, the Maurer-Cartan form series takes the form

$$
\mu+\mu_{X} H+\frac{1}{2} \mu_{X X} H^{2}+\cdots=\mu \llbracket H \rrbracket=\Upsilon \llbracket h \rrbracket=\Upsilon+\Upsilon_{x} h+\frac{1}{2} \Upsilon_{x x} h^{2}+\cdots
$$

when

$$
H=X \llbracket h \rrbracket-X \llbracket 0 \rrbracket=X_{x} h+\frac{1}{2} X_{x x} h^{2}+\frac{1}{6} X_{x x x} h^{3}+\cdots .
$$

Our previous formulae (3.13) for the Maurer-Cartan forms $\mu_{n}=\mathbb{D}_{X}^{n} \mu$ on $\mathcal{D}^{(\infty)}(\mathbb{R})$ result from equating successive powers of $h$ in the expansion (4.12). The diffeomorphism structure equations (4.10), (4.11) are given in the form

$$
d \sigma=\mu_{X} \wedge \sigma, \quad d \mu \llbracket H \rrbracket=\nabla_{H} \mu \llbracket H \rrbracket \wedge(\mu \llbracket H \rrbracket-d Z \llbracket 0 \rrbracket),
$$

where

$$
\nabla_{H} \mu \llbracket H \rrbracket=\mu_{X}+\mu_{X X} H+\frac{1}{2} \mu_{X X X} H^{2}+\cdots
$$

is merely the derivative of the Maurer-Cartan form series $\mu \llbracket H \rrbracket$ with respect to $H$, while

$$
\mu \llbracket H \rrbracket-d Z \llbracket 0 \rrbracket=-\sigma+\mu_{X} H+\frac{1}{2} \mu_{X X} H^{2}+\cdots .
$$

The individual components satisfy

$$
\begin{aligned}
d \sigma & =\mu_{1} \wedge \sigma \\
d \mu_{n} & =-\mu_{n+1} \wedge \sigma+\sum_{i=0}^{n-1}\left(\begin{array}{c}
n \\
i
\end{array}\right) \mu_{i+1} \wedge \mu_{n-i} \\
& =\sigma \wedge \mu_{n+1}-\sum_{j=1}^{[(n+1) / 2]} \frac{n-2 j+1}{n+1}\left(\begin{array}{c}
n+1 \\
j
\end{array}\right) \mu_{j} \wedge \mu_{n+1-j} .
\end{aligned}
$$

In this manner, we recover the general formulae established by Cartan, [13; eq. (48)], for the structure equations for the one-dimensional diffeomorphism pseudo-group $\mathcal{D}(R)$.

Example 4.2. The Maurer-Cartan forms for the planar diffeomorphism pseudogroup $\mathcal{D}\left(\mathbb{R}^{2}\right)$ are obtained as follows. Using the coordinates of Example 2.3, we begin with the zero $^{\text {th }}$ order right-invariant forms

$$
\begin{array}{lll}
\sigma=d_{M} X=X_{x} d x+X_{u} d u, & \tau=d_{M} U=U_{x} d x+U_{u} d u, \\
\mu=\Upsilon=d_{G} X=d X-X_{x} d x-X_{u} d u, & \nu=\Psi=d_{G} U=d U-U_{x} d x-U_{u} d u .
\end{array}
$$


The higher-order right-invariant contact forms are obtained by repeatedly applying the right-invariant differential operators

$$
\mathbb{D}_{X}=\frac{U_{u} \mathbb{D}_{x}-U_{x} \mathbb{D}_{u}}{X_{x} U_{u}-X_{u} U_{x}}, \quad \mathbb{D}_{U}=\frac{-X_{u} \mathbb{D}_{x}+X_{x} \mathbb{D}_{u}}{X_{x} U_{u}-X_{u} U_{x}},
$$

to $\mu, \nu$. In particular, the first order Maurer-Cartan forms are

$$
\begin{aligned}
\mu_{X} & =\frac{U_{u} \Upsilon_{x}-U_{x} \Upsilon_{u}}{X_{x} U_{u}-X_{u} U_{x}}, & \mu_{U} & =\frac{X_{x} \Upsilon_{u}-X_{u} \Upsilon_{x}}{X_{x} U_{u}-X_{u} U_{x}}, \\
\nu_{X} & =\frac{U_{u} \Psi_{x}-U_{x} \Psi_{u}}{X_{x} U_{u}-X_{u} U_{x}}, & \nu_{U} & =\frac{X_{x} \Psi_{u}-X_{u} \Psi_{x}}{X_{x} U_{u}-X_{u} U_{x}},
\end{aligned}
$$

where

$$
\begin{array}{ll}
\Upsilon_{x}=d X_{x}-X_{x x} d x-X_{x u} d u, & \Upsilon_{u}=d X_{u}-X_{x u} d x-X_{u u} d u \\
\Psi_{x}=d U_{x}-U_{x x} d x-U_{x u} d u, & \Psi_{u}=d U_{u}-U_{x u} d x-U_{u u} d u
\end{array}
$$

are the first order contact forms on $\mathcal{D}^{(\infty)}\left(\mathbb{R}^{2}\right)$. The structure equations are found via the general power series formula (4.10), which can be written in the matrix form

$$
\left(\begin{array}{c}
d \mu \llbracket H, K \rrbracket \\
d \nu \llbracket H, K \rrbracket
\end{array}\right)=\left(\begin{array}{cc}
\mu_{H} \llbracket H, K \rrbracket & \mu_{K} \llbracket H, K \rrbracket \\
\nu_{H} \llbracket H, K \rrbracket & \nu_{K} \llbracket H, K \rrbracket
\end{array}\right) \wedge\left(\begin{array}{c}
\mu \llbracket H, K \rrbracket-d X \\
\nu \llbracket H, K \rrbracket-d U
\end{array}\right),
$$

where

$$
\begin{array}{ll}
\mu \llbracket H, K \rrbracket=\sum_{j, k \geq 0} \frac{1}{j ! k !} \mu_{j, k} H^{j} K^{k}, \quad \mu \llbracket H, K \rrbracket-d X=-\sigma+\sum_{\substack{j+k \geq 1 \\
j, k \geq 0}} \frac{1}{j ! k !} \mu_{j, k} H^{j} K^{k}, \\
\nu \llbracket H, K \rrbracket=\sum_{j, k \geq 0} \frac{1}{j ! k !} \nu_{j, k} H^{j} K^{k}, \quad \nu \llbracket H, K \rrbracket-d U=-\tau+\sum_{\substack{j+k \geq 1 \\
j, k \geq 0}} \frac{1}{j ! k !} \nu_{j, k} H^{j} K^{k},
\end{array}
$$

with $\mu_{j, k}=\mathbb{D}_{X}^{j} \mathbb{D}_{U}^{k} \mu, \nu_{j, k}=\mathbb{D}_{X}^{j} \mathbb{D}_{U}^{k} \nu$. In particular, the first order structure equations reduce to

$$
\begin{aligned}
& d \mu_{X}=-\mu_{X X} \wedge \sigma-\mu_{X U} \wedge \tau+\mu_{U} \wedge \nu_{X}, \\
& d \sigma=-d \mu=\mu_{X} \wedge \sigma+\mu_{U} \wedge \tau, \quad \\
& d \nu_{X}=-\nu_{X X} \wedge \sigma-\nu_{X U} \wedge \tau+\nu_{X} \wedge\left(\mu_{X}-\nu_{U}\right), \\
& d \mu_{U}=-\mu_{X U} \wedge \sigma-\mu_{U U} \wedge \tau-\mu_{U} \wedge\left(\mu_{X}-\nu_{U}\right), \\
& d \nu_{U}=-\nu_{X U} \wedge \sigma-\nu_{U U} \wedge \tau+\nu_{X} \wedge \mu_{U}
\end{aligned}
$$

\section{Infinitesimal Generators.}

Let $\mathcal{X}=\mathcal{X}(M)$ denote the space of locally defined vector fields on $M$, i.e., local sections of the tangent bundle $T M$. Let $\mathrm{J}^{n} T M=\mathrm{J}^{n} T M, 0 \leq n \leq \infty$, denote the tangent $n$-jet bundle. Let $\mathcal{A}^{(n)}$ denote the Lie algebroid, [47], of the jet groupoid $\mathcal{D}^{(n)}$ which is, by definition, the space of right-invariant vector fields that are tangent to the source 
fibers $\left.\mathcal{D}^{(n)}\right|_{z}$. Each Lie algebroid vector field is uniquely specified by its value on the identity section $\mathbb{1}^{(n)}$, and so we can identify $\mathcal{A}^{(n)}$ as the space of sections of the vector bundle $\mathcal{R}^{(n)}=\left.\bigcup_{z \in M} T\left(\left.\mathcal{D}^{(n)}\right|_{z}\right)\right|_{\mathbb{1}_{z}^{(n)}} \rightarrow M$. There is a natural Lie algebra isomorphism $\boldsymbol{\lambda}^{(n)}: \mathcal{X}(M) \simeq \mathcal{A}^{(n)}$ that maps the infinitesimal generator $\mathbf{v}$ of a local flow $\Phi_{t}$ acting on $M$ to the infinitesimal generator $\mathbf{V}^{(n)}=\boldsymbol{\lambda}^{(n)}(\mathbf{v})$ of the flow induced by the left action of $\Phi_{t}$ on $\mathcal{D}^{(n)}$. We will call $\mathbf{V}^{(n)}$ the $n^{\text {th }}$ order lift of the vector field $\mathbf{v}$. The infinite order case will be simply denoted by $\mathbf{V}=\boldsymbol{\lambda}(\mathbf{v})$, and the finite order cases are obtained by truncation.

In local coordinates, the lift of a vector field

$$
\mathbf{v}=\sum_{a=1}^{m} \zeta^{a}(z) \frac{\partial}{\partial z^{a}}
$$

on $M$ is the right-invariant vector field

$$
\mathbf{V}=\sum_{a=1}^{m} \sum_{\# J \geq 0} \mathbb{D}_{z}^{J} \zeta^{a}(Z) \frac{\partial}{\partial Z_{J}^{a}}
$$

on $\mathcal{D}^{(\infty)}$, in which one replaces the source variables $z$ by target variables $Z$ in the vector field coefficients, and then differentiates with respect to $z$ via the chain rule using the differential operators

$$
\mathbb{D}_{z^{i}}=\sum_{a=1}^{m} Z_{i}^{a} \mathbb{D}_{Z^{a}}, \quad i=1, \ldots, m .
$$

Thus, lifting essentially coincides with prolongation of a vertical vector field on $M \times M$ to the jet bundle $\mathrm{J}^{n}(M, M)$, cf. [55].

Example 5.1. In the case $M=\mathbb{R}$, the lift of a vector field $\mathbf{v}=\xi(x) \partial_{x}$ is obtained by prolonging the associated vertical vector field $\xi(X) \partial_{X}$ to $\mathcal{D}^{(\infty)}$, and so

$$
\begin{aligned}
\mathbf{V}=\xi & \frac{\partial}{\partial X}+X_{x} \xi_{X} \frac{\partial}{\partial X_{x}}+\left(X_{x}^{2} \xi_{X X}+X_{x x} \xi_{X}\right) \frac{\partial}{\partial X_{x x}}+ \\
& +\left(X_{x}^{3} \xi_{X X X}+3 X_{x} X_{x x} \xi_{X X}+X_{x x x} \xi_{X}\right) \frac{\partial}{\partial X_{x x x}}+\cdots
\end{aligned}
$$

On the level of jets, we can view $\xi, \xi_{X}, \xi_{X X}, \ldots$ as independent jet coordinates, and hence (5.4) is a linear combination of the basis Lie algebroid generators

$$
\begin{array}{ccc}
\frac{\partial}{\partial X}, & X_{x} \frac{\partial}{\partial X_{x}}+X_{x x} \frac{\partial}{\partial X_{x x}}+X_{x x x} \frac{\partial}{\partial X_{x x x}}+\cdots, \\
X_{x}^{2} \frac{\partial}{\partial X_{x x}}+3 X_{x} X_{x x} \frac{\partial}{\partial X_{x x x}}+\cdots, & X_{x}^{3} \frac{\partial}{\partial X_{x x x}}+\cdots
\end{array}
$$

and so on. The general formula can be explicitly written in terms of Bell polynomials, $[59,65]$. The dual basis for the Lie algebroid $\mathcal{A}^{(n)}$ relative to the basis Maurer-Cartan forms constructed above is obtained by truncating the expansions at order $n$. 
Let $\mathcal{Z}^{(n)}$ denote the dual bundle to the vector field jet bundle $\mathrm{J}^{n} T M$, and $\mathcal{Z}^{(\infty)}$ the direct limit. (Warning: $\mathcal{Z}^{(n)}$ is not the same as the space of jets of differential one-forms on $M$.) We define the lift of a section $\zeta$ of $\mathcal{Z}^{(\infty)}$ to be the right-invariant differential form $\boldsymbol{\lambda}(\zeta)$ on $\mathcal{D}^{(\infty)}$ that vanishes on all total vector fields, and satisfies

$$
\left.\langle\boldsymbol{\lambda}(\zeta) ; \boldsymbol{\lambda}(\mathbf{v})\rangle\right|_{g^{(\infty)}}=\left.\left\langle\zeta ; j_{\infty} \mathbf{v}\right\rangle\right|_{Z} \quad \text { whenever } \quad \begin{array}{ll}
(\infty) \in \mathcal{D}^{(\infty)}, \\
& Z=\boldsymbol{\tau}^{(\infty)}\left(g^{(\infty)}\right) .
\end{array}
$$

In local coordinates, each vector field jet coordinate function $\zeta_{J}^{a}$ can be viewed as a section of $\mathcal{Z}^{(\infty)}$, and is easily shown to lift to the corresponding basic right-invariant contact form

$$
\mu_{J}^{a}=\boldsymbol{\lambda}\left(\zeta_{J}^{a}\right)
$$

More generally, any linear function of the vector field jets, $L\left(z, \zeta^{(n)}\right)$, can be viewed as a section of $\mathcal{Z}^{(n)}$, whose lift

$$
\boldsymbol{\lambda}\left[L\left(z, \zeta^{(n)}\right)\right]=L\left(Z, \mu^{(n)}\right)
$$

is formally obtained by replacing the source variables $z^{a}$ by their target counterparts $Z^{a}$ and the vector field jet coordinates $\zeta_{J}^{a}$ by the Maurer-Cartan forms $\mu_{J}^{a}$.

Given a pseudo-group $\mathcal{G}$, let $\mathfrak{g} \subset \mathcal{X}$ denote the local Lie algebra of infinitesimal generators, i.e., the set of locally defined vector fields whose flows belong to the pseudogroup. Let $\mathrm{J}^{n} \mathfrak{g} \subset \mathrm{J}^{n} T M$ denote their jets. In local coordinates, we can view the subbundle $\mathrm{J}^{n} \mathfrak{g} \subset \mathrm{J}^{n} T M$ as defining a linear system of partial differential equations

$$
L^{(n)}\left(z, \zeta^{(n)}\right)=0
$$

for the vector field coefficients, called the linearized or infinitesimal determining equations for the pseudo-group. In practice, they are constructed by linearizing the $n^{\text {th }}$ order determining equations (2.7) at the identity:

$$
L^{(n)}\left(z, \zeta^{(n)}\right)=\left.\mathbf{V}^{(n)}\left[F^{(n)}\left(z, Z^{(n)}\right)\right]\right|_{\left(z, Z^{(n)}\right)=\mathbb{1}_{z}^{(n)}} .
$$

The infinitesimal determining equations form a linear Lie equation, cf. [40], meaning that the Lie bracket of any two solutions is again a solution.

Remark: If $\mathcal{G}$ is the symmetry group of a system of differential equations, then the linearized determining equations (5.8) are the (involutive completion of) the usual determining equations for its infinitesimal generators obtained via Lie's algorithm, $[\mathbf{4 5}, \mathbf{4 6}, \mathbf{5 4}]$. Infinite-dimensional pseudo-groups arise as symmetry groups of a broad range of systems, including solitons, [19], fluid mechanics, [54], oceanography and meteorology, [69], and gauge theory, $[6]$.

By the preceding remarks, for $n \geq n^{\star}$, the general solution to the linearized determining equations (5.8) is precisely the space of infinitesimal generators $\mathfrak{g}$. The system is formally integrable by virtue of condition $(b)$ of Definition 2.2. Under reasonable hypotheses, involutivity should follow from the involutivity of the nonlinear determining equations (2.7), although a precise statement seems tricky, $[\mathbf{4 1}, \mathbf{4 2}]$. However, local solvability of the infinitesimal determining equations does not seem to follow from our definitions so far, and so we make the following additional mild restriction. 
First, the Lie algebroid $\mathcal{L}^{(n)}$ of the jet groupoid $\mathcal{G}^{(n)}$ is the set of right-invariant vector fields on $\mathcal{G}^{(n)}$ tangent to the source fibers. A Lie algebroid vector field is uniquely determined by its value at the identity section, and hence there is a one-to-one correspondence between Lie algebroid elements and sections of $\mathcal{W}^{(n)}=\left.\bigcup_{z \in M} T\left(\left.\mathcal{G}^{(n)}\right|_{z}\right)\right|_{\mathbb{1}_{z}^{(n)}}$, which, under the hypotheses of Definition 2.2 is a vector subbundle $\mathcal{W}^{(n)} \subset \mathcal{R}^{(n)}$ for all $n \geq n^{\star}$.

Definition 5.2. A pseudo-group $\mathcal{G} \subset \mathcal{D}$ is tame if for all $z \in M$ and all $n \geq n^{\star}$, each $\left.\mathbf{w} \in \mathcal{W}^{(n)}\right|_{z}$ is the lift of some $\mathbf{v} \in \mathfrak{g}$, that is, $\left.\boldsymbol{\lambda}^{(n)}(\mathbf{v})\right|_{\mathbb{1}_{z}^{(n)}}=\mathbf{w}$.

Remark: All known (at least to us) examples satisfy the tameness condition, but we do not know if it is valid for all pseudo-groups satisfying the hypotheses of Definition 2.2. From now on, all pseudo-groups are assumed to be tame.

Example 5.3. The infinitesimal generators of the pseudo-group given in Example 2.3 are all vector fields of the form

$$
\mathbf{v}=\xi \frac{\partial}{\partial x}+\varphi \frac{\partial}{\partial u}=a(x) \frac{\partial}{\partial x}-a^{\prime}(x) u \frac{\partial}{\partial u},
$$

where $a(x)$ is an arbitrary smooth function. The coefficients $\xi=a(x)$ and $\varphi=-u a^{\prime}(x)$ form the general solution to the first order infinitesimal determining system

$$
\xi_{x}=-\frac{\varphi}{u}, \quad \xi_{u}=0, \quad \varphi_{u}=\frac{\varphi}{u},
$$

which is obtained by linearizing the determining system (2.11) at the identity, i.e., where $X=x, \quad U=u, \quad X_{x}=U_{u}=1, \quad X_{u}=X_{x x}=X_{x u}=\cdots=U_{x}=U_{x x}=U_{x u}=\cdots=0$.

The system (5.11) is involutive, and hence the higher-order infinitesimal determining systems are obtained by repeatedly applying the total derivative operators

$$
\begin{aligned}
& \mathbb{D}_{x}=\frac{\partial}{\partial x}+\xi_{x} \frac{\partial}{\partial \xi}+\varphi_{x} \frac{\partial}{\partial \varphi}+\xi_{x x} \frac{\partial}{\partial \xi_{x}}+\xi_{x u} \frac{\partial}{\partial \xi_{u}}+\varphi_{x x} \frac{\partial}{\partial \varphi_{x}}+\varphi_{x u} \frac{\partial}{\partial \varphi_{u}}+\cdots \\
& \mathbb{D}_{u}=\frac{\partial}{\partial u}+\xi_{u} \frac{\partial}{\partial \xi}+\varphi_{u} \frac{\partial}{\partial \varphi}+\xi_{x u} \frac{\partial}{\partial \xi_{x}}+\xi_{u u} \frac{\partial}{\partial \xi_{u}}+\varphi_{x u} \frac{\partial}{\partial \varphi_{x}}+\varphi_{u u} \frac{\partial}{\partial \varphi_{u}}+\cdots
\end{aligned}
$$

Example 5.4. Similarly, the infinitesimal generators of the pseudo-group (2.14) are

$$
\mathbf{v}=\xi \frac{\partial}{\partial x}+\eta \frac{\partial}{\partial y}+\varphi \frac{\partial}{\partial u}=a(x) \frac{\partial}{\partial x}+\left[a^{\prime}(x) y+b(x)\right] \frac{\partial}{\partial y}+\left[a^{\prime \prime}(x) y+b^{\prime}(x)\right] \frac{\partial}{\partial u},
$$

where $a(x), b(x)$ are arbitrary smooth functions. These form the general solution to the first order infinitesimal determining system

$$
\xi_{x}=\eta_{y}, \quad \xi_{y}=\xi_{u}=\eta_{u}=\varphi_{u}=0, \quad \eta_{x}=\varphi,
$$

which is obtained by linearizing the determining system (2.15) at the identity jet. Again, the linearized determining equations are involutive at order 1. 


\section{The Structure Equations for a Pseudo-Group.}

A complete system of right-invariant one-forms on the infinite jet groupoid $\mathcal{G}^{(\infty)} \subset$ $\mathcal{D}^{(\infty)}$ is obtained by restricting (pulling back) the Maurer-Cartan forms (3.6), (3.10). For simplicity, we continue to denote these forms by $\sigma=\left(\ldots \sigma^{a} \ldots\right), \mu^{(\infty)}=\left(\ldots \mu_{J}^{a} \ldots\right)$. The restricted Maurer-Cartan forms are, of course, no longer linearly independent, but are subject to the constraints prescribed by the pseudo-group. Remarkably, these constraints can be explicitly characterized by an invariant version of the linearized determining equations (5.8). The proof, though, is a simple unwinding of definitions.

Theorem 6.1. The linear system

$$
L^{(n)}\left(Z, \mu^{(n)}\right)=0,
$$

obtained by lifting the linear determining equations (5.8) as in (5.7) serves to define the complete set of linear dependencies among the right-invariant Maurer-Cartan forms $\mu^{(n)}$ on $\mathcal{G}^{(n)}$.

Proof: At each point $z \in M$, the linear determining equations are defined by a system of linear functions on the vector field jet bundle $\mathrm{J}^{n} T M$, which we can view locally as a collection of sections of the dual bundle $\mathcal{Z}^{(n)}$. Their lift, as in (5.7), forms a system of rightinvariant forms on the diffeomorphism groupoid. Since the linear determining equations annihilate the tangent spaces $\left.T\left(\left.\mathcal{G}^{(n)}\right|_{z}\right)\right|_{\mathbb{1}_{z}^{(n)}}$, formula (5.5) immediately implies that the tangent space to $\mathcal{G}^{(n)}$ is the annihilator of the lifted system of differential forms, and hence (6.1) follows.

Q.E.D.

Corollary 6.2. The structure equations for the pseudo-group are obtained by restriction of the diffeomorphism structure equations (4.10) to the kernel of the linearized involutive system, as defined by (6.1).

An invariant coframe on the infinite pseudo-group jet bundle $\mathcal{G}^{(\infty)}$ can be identified as a basis for the solution space to the infinite prolongation of the linear system (6.1). Under appropriate hypotheses, truncating at the order of involutivity of the linearized determining system (5.8) yields an involutive Pfaffian system that serves to characterize the pseudo-group.

Example 6.3. We first illustrate the method by treating a standard finite-dimensional Lie group action, also analyzed by Cartan, [14]. The linear fractional transformations $X=(\alpha x+\beta) /(\gamma x+\delta)$ define an action of the projective group $\operatorname{PGL}(2, \mathbb{R})$ on $M=\mathbb{R P}^{1}$. The determining equations are obtained by prolonging the well-known Schwarzian equation

$$
X_{x x x} X_{x}-\frac{3}{2} X_{x x}^{2}=0 .
$$

The infinitesimal generators $\mathbf{v}=\xi(x) \partial_{x}=\left(a+b x+c x^{2}\right) \partial_{x}$ solve the linear determining equation

$$
\xi_{x x x}=0
$$

obtained by linearizing (6.2) at the identity $X=x, X_{x}=1, X_{x x}=X_{x x x}=\cdots=0$. 
The Maurer-Cartan forms on the jet groupoid $\mathcal{G}^{(\infty)} \subset \mathcal{D}^{(\infty)}$ are obtained by restriction of the right-invariant coframe $\sigma, \mu, \mu_{X}, \mu_{X X}, \ldots$ from Example 3.2 to the group manifold, as defined by (6.2) and its prolongations. According to Theorem 6.1, they satisfy

$$
0=\mu_{X X X}=\mu_{X X X X}=\cdots
$$

which can be verified by direct computation. Therefore, referring to (3.13), the one-forms

$$
\begin{gathered}
\sigma=X_{x} d x, \quad \mu=d X-X_{x} d x, \quad \mu_{X}=\frac{\Upsilon_{x}}{X_{x}}=\frac{d X_{x}-X_{x x} d x}{X_{x}} \\
\mu_{X X}=\frac{X_{x} \Upsilon_{x x}-X_{x x} \Upsilon_{x}}{X_{x}^{3}}=\frac{X_{x} d X_{x x}-X_{x x} d X_{x}-\frac{1}{2} X_{x x}^{2} d x}{X_{x}^{3}}
\end{gathered}
$$

provide a right-invariant coframe on $\mathcal{G}^{(2)} \simeq \mathcal{G}^{(\infty)}$, with local coordinates $x, X, X_{x}, X_{x x}$. The structure equations are found by truncating (4.14) and invoking (6.3):

$$
d \sigma=-d \mu=\mu_{X} \wedge \sigma, \quad d \mu_{X}=\sigma \wedge \mu_{X X}, \quad d \mu_{X X}=-\mu_{X} \wedge \mu_{X X},
$$

and so the one-forms $\sigma, \mu_{X}, \mu_{X X}$ satisfy the structure equations of $\operatorname{PGL}(2, \mathbb{R})$. The additional one-form $\mu$ arises from the fact that $\mathcal{G}^{(2)}$ can be identified as a principal $\operatorname{PGL}(2, \mathbb{R})$ bundle over $\mathbb{R P}^{1}$. Indeed, by transitivity, each target fiber $\left(\boldsymbol{\tau}^{(2)}\right)^{-1}\{c\}=\{X=c\} \subset \mathcal{G}^{(2)}$ can be identified with $\operatorname{PGL}(2, \mathbb{R})$. Since $\sigma+\mu=d X=0$ on the target fibers, the restriction of the invariant one-forms yields a complete system of (fiber-dependent linear combinations of) the standard Maurer-Cartan coframe for $\operatorname{PGL}(2, \mathbb{R})$.

Example 6.4. The Maurer-Cartan forms for the pseudo-group (2.9) are obtained by restricting the right-invariant forms $(4.15)$, (4.17) to the subbundle defined by the pseudo-group determining equations $(2.11),(2.13)$. The basic invariant one-forms are

$$
\begin{aligned}
\sigma & =\frac{u}{U} d x=f_{x} d x, & \tau & =U_{x} d x+\frac{U}{u} d u=\frac{-u f_{x x} d x+f_{x} d u}{f_{x}^{2}}, \\
\mu & =d X-\frac{u}{U} d x=\Phi, & \nu & =d U-U_{x} d x-\frac{U}{u} d u=-\frac{u \Phi_{x}}{f_{x}^{2}},
\end{aligned}
$$

where

$$
\Phi=d f-f_{x} d x, \quad \Phi_{x}=d f_{x}-f_{x x} d x, \quad \ldots \quad \Phi_{n}=d f_{n}-f_{n+1} d x,
$$

are contact forms in the pseudo-group parameters. The higher-order Maurer-Cartan forms are obtained by similarly restricting the higher-order diffeomorphism Maurer-Cartan forms $\left(\sigma, \mu^{(\infty)}\right)$, or, alternatively, by successively differentiating $\mu, \nu$ using the dual total differential operators

$$
\mathbb{D}_{X}=\frac{U}{u} \mathbb{D}_{x}-U_{x} \mathbb{D}_{u}=\frac{1}{f_{x}} \mathbb{D}_{x}+\frac{u f_{x x}}{f_{x}^{2}} \mathbb{D}_{u}, \quad \mathbb{D}_{U}=\frac{u}{U} \mathbb{D}_{u}=f_{x} \mathbb{D}_{u}
$$

where, on the pseudo-group bundle,

$$
\mathbb{D}_{x}=\frac{\partial}{\partial x}+\sum_{i \geq 0} f_{i+1} \frac{\partial}{\partial f_{i}}, \quad \mathbb{D}_{u}=\frac{\partial}{\partial u} .
$$


Thus, the first order Maurer-Cartan forms are

$$
\begin{aligned}
& \mu_{X}=\frac{d u}{u}-\frac{d U-U_{x} d x}{U}=\frac{\Phi_{x}}{f_{x}}, \quad \nu_{U}=-\frac{d u}{u}+\frac{d U-U_{x} d x}{U}=-\frac{\Phi_{x}}{f_{x}}, \\
& \mu_{U}=0, \quad \nu_{X}=\frac{U\left(d U_{x}-U_{x x} d x\right)-U_{x}\left(d U-U_{x} d x\right)}{u}=\frac{u f_{x x} \Phi_{x}-u f_{x} \Phi_{x x}}{f_{x}^{4}} .
\end{aligned}
$$

Note that, when written in terms of the parameters $f, f_{x}, f_{x x}$, the Maurer-Cartan forms are certain linear combinations

$$
\mu, \quad \nu=-U \mu_{X}, \quad \mu_{X}, \quad \mu_{U}=0, \quad \nu_{X}=-U \mu_{X X}, \quad \nu_{U}=-\mu_{X},
$$

of the Maurer-Cartan forms (3.13) for the diffeomorphism group $\mathcal{D}(\mathbb{R})$. In Cartan's terminology, the pseudo-group is a "holohedric prolongation" of $\mathcal{D}(\mathbb{R})$, and this correspondence is maintained on the level of their Maurer-Cartan forms.

In accordance with Theorem 6.1, the Maurer-Cartan forms (6.6), (6.8) are constrained by the invariantized version

$$
\mu_{X}=-\frac{\nu}{U}, \quad \mu_{U}=0, \quad \nu_{U}=\frac{\nu}{U},
$$

of the linear determining equations (5.11). The higher-order constraints are obtained by (invariant) prolongation - i.e., differentiation of (6.10) with respect to $X, U$. Thus, at second order

$$
\mu_{X X}=-\nu_{X U}=-\frac{\nu_{X}}{U}, \quad \mu_{X U}=\mu_{U U}=\nu_{U U}=0 .
$$

The remaining independent one-forms $\sigma, \tau, \mu, \nu, \nu_{X}, \nu_{X X}, \nu_{X X X}, \ldots$ form a right-invariant coframe on $\mathcal{G}^{(\infty)}$. The structure equations (at order 1 )

$$
\begin{array}{ll}
d \sigma=-d \mu=-\frac{\nu \wedge \sigma}{U}, & d \mu_{X}=\frac{\nu_{X} \wedge \sigma}{U}, \\
d \tau=-d \nu=\nu_{X} \wedge \sigma+\frac{\nu \wedge \tau}{U}, & d \nu_{X}=-\nu_{X X} \wedge \sigma-\frac{\nu_{X} \wedge(\tau+2 \nu)}{U},
\end{array}
$$

are obtained by restriction of the structure equations (4.19). Higher order structure equations can be obtained from the general power series expansion. Indeed, the solution to the invariant determining equations (6.10) can be written in terms of the $\mathcal{D}(\mathbb{R})$ Maurer-Cartan form power series (4.12):

$$
\mu \llbracket H, K \rrbracket=\mu \llbracket H \rrbracket, \quad \nu \llbracket H, K \rrbracket=-(U+K) \mu_{H} \llbracket H \rrbracket .
$$

Substituting (6.12) reduces the structure equations (4.18) to the structure equations (4.14) for $\mathcal{D}(\mathbb{R})$, in accordance with the holohedric equivalence of the two pseudo-groups.

Example 6.5. The Maurer-Cartan forms for the pseudo-group (2.14) are obtained as follows. To simplify the final expressions, we use $f(x)$ and

$$
e(x, y)=f^{\prime}(x) y+g(x)
$$


to denote the arbitrary functions, which are subject to the constraints $e_{y}=f_{x}, f_{y}=0$. For all $n \geq 1$, a complete system of local coordinates on $\mathcal{G}^{(n)}$ is given by $\left(x, y, u, f, e, f_{x}, e_{x}, \ldots\right.$, $\left.f_{n+1}, e_{n+1}\right)$, where

$$
f_{k}=\partial_{x}^{k} f, \quad e_{k}=\partial_{x}^{k} e=y \partial_{x}^{k+1} f+\partial_{x}^{k} g, \quad k \geq 0 .
$$

The basic invariant horizontal forms are

$$
\begin{gathered}
\sigma=d_{M} X=f_{x} d x, \quad \tilde{\sigma}=d_{M} Y=f_{x} d y+e_{x} d x, \\
\tau=d_{M} U=d u+\frac{\left(f_{x} e_{x x}-f_{x x} e_{x}\right) d x}{f_{x}^{2}}+\frac{f_{x x} d y}{f_{x}} .
\end{gathered}
$$

The order zero invariant contact forms are

$$
\mu=d_{G} X=\Phi, \quad \tilde{\mu}=d_{G} Y=\Psi, \quad \nu=d_{G} U=\frac{f_{x} \Psi_{x}-e_{x} \Phi_{x}}{f_{x}^{2}},
$$

where $\Phi, \Phi_{x}$ are given in (6.7), while

$$
\begin{gathered}
\Psi=d e-e_{x} d x-f_{x} d y, \quad \Psi_{x}=d e_{x}-e_{x x} d x-f_{x x} d y, \quad \ldots \\
\Psi_{n}=d e_{n}-e_{n+1} d x-f_{n+1} d y .
\end{gathered}
$$

The invariant differential operators dual to (6.14) are given by

$$
\mathbb{D}_{X}=\frac{1}{f_{x}} \mathbb{D}_{x}-\frac{e_{x}}{f_{x}^{2}} \mathbb{D}_{y}+\frac{2 f_{x x} e_{x}-f_{x} e_{x x}}{f_{x}^{3}} \mathbb{D}_{u}, \quad \mathbb{D}_{Y}=\frac{1}{f_{x}} \mathbb{D}_{y}-\frac{f_{x x}}{f_{x}^{2}} \mathbb{D}_{u}, \quad \mathbb{D}_{U}=\mathbb{D}_{u}
$$

where, when written in terms of the pseudo-group parameters,

$$
\mathbb{D}_{x}=\frac{\partial}{\partial x}+\sum_{n=0}^{\infty}\left(f_{n+1} \frac{\partial}{\partial f_{n}}+e_{n+1} \frac{\partial}{\partial e_{n}}\right), \quad \mathbb{D}_{y}=\frac{\partial}{\partial y}+\sum_{n=0}^{\infty} f_{n+1} \frac{\partial}{\partial e_{n}}, \quad \mathbb{D}_{u}=\frac{\partial}{\partial u}
$$

Applying the differential operators (6.17) repeatedly to the order zero forms (6.15) produces the higher-order Maurer-Cartan forms

$$
\begin{array}{ll}
\mu_{Y}=\mu_{U}=\widetilde{\mu}_{U}=\nu_{U}=0, \quad \mu_{X}=\widetilde{\mu}_{Y}=\frac{\Phi_{x}}{f_{x}}, & \widetilde{\mu}_{X}=\frac{f_{x} \Psi_{x}-e_{x} \Phi_{x}}{f_{x}^{2}}, \\
\nu_{X}=\frac{f_{x}^{2} \Psi_{x x}-f_{x} f_{x x} \Psi_{x}-2 f_{x} e_{x} \Phi_{x x}+\left(3 e_{x} f_{x x}-e_{x x} f_{x}\right) \Phi_{x}}{f_{x}^{4}}, & \nu_{Y}=\frac{f_{x} \Phi_{x x}-f_{x x} \Phi_{x}}{f_{x}^{3}}
\end{array}
$$

and so on. In accordance with Theorem 6.1, the contact Maurer-Cartan forms satisfy the invariant linearized determining equations for the pseudo-group $\mathcal{G}$; to first order,

$$
\mu_{X}=\widetilde{\mu}_{Y}, \quad \mu_{Y}=\mu_{U}=\widetilde{\mu}_{U}=\nu_{U}=0, \quad \widetilde{\mu}_{X}=\nu,
$$

By repeated differentiation, setting $\mu_{j, k, l}=\mathbb{D}_{X}^{j} \mathbb{D}_{Y}^{k} \mathbb{D}_{U}^{l} \mu$, etc., we find that

$$
\widetilde{\mu}_{j, 1,0}=\mu_{j+1,0,0}, \quad \nu_{j, 0,0}=\widetilde{\mu}_{j+1,0,0}, \quad \nu_{j, 1,0}=\mu_{j+2,0,0}, \quad j, k, l \geq 0
$$


and all others are zero. As a result, the one-forms

$$
\sigma, \quad \tilde{\sigma}, \quad \tau, \quad \mu_{n}=\mu_{n, 0,0}, \quad \tilde{\mu}_{n}=\tilde{\mu}_{n, 0,0} \quad \text { for } \quad n=0,1,2, \ldots,
$$

form a $\mathcal{G}$-invariant coframe on $\mathcal{G}^{(\infty)}$.

The structure equations are obtained by substituting the expansions

$$
\mu \llbracket H \rrbracket=\sum_{n=0}^{\infty} \frac{1}{n !} \mu_{n} H^{n}
$$

$$
\begin{aligned}
& \widetilde{\mu} \llbracket H, K \rrbracket=\sum_{n=0}^{\infty} \frac{1}{n !} \widetilde{\mu}_{n} H^{n}+K \sum_{n=0}^{\infty} \frac{1}{n !} \mu_{n+1} H^{n}, \\
& \nu \llbracket H, K \rrbracket=\sum_{n=0}^{\infty} \frac{1}{n !} \widetilde{\mu}_{n+1} H^{n}+K \sum_{n=0}^{\infty} \frac{1}{n !} \mu_{n+2} H^{n},
\end{aligned}
$$

into (4.10). Those involving $\mu_{n}$ reduce to the structure equations (4.14) for $\mathcal{D}(R)$ while

$$
d \widetilde{\mu}_{n}=\sigma \wedge \widetilde{\mu}_{n+1}+\widetilde{\sigma} \wedge \mu_{n+1}+\sum_{j=0}^{n-1}\left[\left(\begin{array}{c}
n \\
j
\end{array}\right)-\left(\begin{array}{c}
n \\
j+1
\end{array}\right)\right] \widetilde{\mu}_{j+1} \wedge \mu_{n-j} .
$$

Moreover,

$$
\begin{aligned}
d \sigma & =-d \mu=-\sigma \wedge \mu_{X}, \\
d \widetilde{\sigma} & =-d \widetilde{\mu}=-\sigma \wedge \widetilde{\mu}_{X}-\widetilde{\sigma} \wedge \mu_{X}, \\
d \tau & =-d \nu=-d \widetilde{\mu}_{X}=-\sigma \wedge \widetilde{\mu}_{X X}-\widetilde{\sigma} \wedge \mu_{X X} .
\end{aligned}
$$

Example 6.6. The following relatively trivial, but nevertheless interesting finitedimensional abelian transformation group

$$
X=x, \quad Y=y, \quad U=u+a x+b y, \quad \text { where } \quad(a, b) \in \mathbb{R}^{2},
$$

appears in Cartan, [14; p. 1357], as an illustration of his theory of essential invariants; see also $[\mathbf{7 2} ;$ p. 407]. The first order determining equations are

$$
X=x, \quad Y=y, \quad U=u+x U_{x}+y U_{y}, \quad X_{x}=Y_{y}=U_{u}=1, \quad X_{y}=X_{u}=Y_{x}=Y_{u}=0 .
$$

The jet coordinates $U_{x}=a, U_{y}=b$, coincide with the group parameters, while all higherorder derivatives are 0 . The invariant horizontal forms are

$$
\sigma=d_{M} X=d x, \quad \tilde{\sigma}=d_{M} Y=d y, \quad \tau=d_{M} U=d u+U_{x} d x+U_{y} d y,
$$

while the non-zero invariant contact forms are

$$
\nu=d_{G} U=x d U_{x}+y d U_{y}, \quad \nu_{X}=d U_{x}, \quad \nu_{Y}=d U_{y} .
$$

They clearly satisfy the infinitesimal determining equation

$$
\nu=X \nu_{X}+Y \nu_{Y}
$$

while all higher-order Maurer-Cartan forms vanish when restricted to $\mathcal{G}^{(\infty)}$. The structure equations reduce to

$$
d \sigma=d \widetilde{\sigma}=d \nu_{X}=d \nu_{Y}=0, \quad d \tau=-d \nu=\nu_{X} \wedge \sigma+\nu_{Y} \wedge \widetilde{\sigma}
$$


However, owing to (6.22), only 5 of the Maurer-Cartan forms are independent, and, on the submanifold where $Y=y \neq 0$, we have

$$
d \tau=-d \nu=\frac{\nu \wedge \widetilde{\sigma}}{Y}+\nu_{X} \wedge\left(\sigma-\frac{X}{Y} \widetilde{\sigma}\right)
$$

reproducing the structure equations found by Cartan. The function $I=X / Y=x / y$ that appears in the last structure equation (6.24) is known as an essential invariant. Essential invariants depend upon the fibration of the jet bundle, and are preserved under equivalence maps on the base manifold. Indeed, the alternative coframe $\sigma, \widetilde{\sigma}, \tau, \nu_{X}, \nu_{Y}$ does not introduce any essential invariant in the structure equations, because the prolonged action on the five-dimensional subbundle $\mathcal{G}^{(1)} \subset \mathcal{D}^{(1)}$ is equivalent, under a non-projectable change of variables, to the trivial translation action of $\mathbb{R}^{2}$ on $\mathbb{R}^{5}$.

Remark: Interestingly, the "enhanced" action $U=u+a x+b y+c$ has no essential invariants; the structure equations are the same, but there is no linear dependence among the Maurer-Cartan forms $\nu, \nu_{X}, \nu_{Y}$ that gives rise to the essential invariant.

The existence of essential invariants does not affect the moving frame algorithm to be presented in [60]. The reader may wish to use Cartan's simple example as a test case.

Acknowledgments: We would like to thank Ian Anderson, Mark Fels, Rui Fernandes, Vladimir Itskov, Irina Kogan, Niky Kamran, Liz Mansfield, and Greg Reid for essential input and advice as this project evolved. The first author would also like to thank John Maddocks and the École Polytechnique Fédérale de Lausanne for their hospitality.

\section{References}

[1] Anderson, I.M., The Variational Bicomplex, Utah State Technical Report, 1989, http://math.usu.edu/ fg_mp.

[2] Anderson, I.M., Introduction to the variational bicomplex, Contemp. Math. 132 (1992), 51-73.

[3] Bazin, P.-L., and Boutin, M., Structure from motion: theoretical foundations of a novel approach using custom built invariants, SIAM J. Appl. Math. 64 (2004), $1156-1174$.

[4] Berchenko, I.A., and Olver, P.J., Symmetries of polynomials, J. Symb. Comp. 29 (2000), 485-514.

[5] Bílă, N., Mansfield, E.L., and Clarkson, P.A., Symmetry group analysis of the shallow water and semi-geostrophic equations, Quart. J. Mech. Appl. Math., to appear.

[6] Bleecker, D., Gauge Theory and Variational Principles, Addison-Wesley Publ. Co., Reading, Mass., 1981.

[7] Boutin, M., Numerically invariant signature curves, Int. J. Computer Vision 40 (2000), 235-248. 
[8] Boutin, M., On orbit dimensions under a simultaneous Lie group action on $n$ copies of a manifold, J. Lie Theory 12 (2002), 191-203.

[9] Calabi, E., Olver, P.J., Shakiban, C., Tannenbaum, A., and Haker, S., Differential and numerically invariant signature curves applied to object recognition, Int. J. Computer Vision 26 (1998), 107-135.

[10] Calmet, C., Hausdorf, M., and Seiler, W.M., A constructive introduction to involution, in: Proc. Int. Symp. Applications of Computer Algebra - ISACA 2000, R. Akerkar (ed), Allied Publishers, New Delhi, 2001, pp. 33-50.

[11] Cantwell, B.J., Introduction to Symmetry Analysis, Cambridge University Press, Cambridge, 2003.

[12] Cartan, É., La Méthode du Repère Mobile, la Théorie des Groupes Continus, et les Espaces Généralisés, Exposés de Géométrie, no. 5, Hermann, Paris, 1935.

[13] Cartan, É., Sur la structure des groupes infinis de transformations, in: Oeuvres Complètes, Part. II, Vol. 2, Gauthier-Villars, Paris, 1953, pp. 571-714.

[14] Cartan, É., La structure des groupes infinis, in: Oeuvres Complètes, part. II, vol. 2, Gauthier-Villars, Paris, 1953, pp. 1335-1384.

[15] Cheh, J., Olver, P.J., and Pohjanpelto, J., Maurer-Cartan equations for Lie symmetry pseudo-groups of differential equations, J. Math. Phys. 46 (2005), 023504.

[16] Cheh, J., Olver, P.J., and Pohjanpelto, J., Differential invariants for Lie symmetry pseudo-groups of differential equations, in preparation.

[17] Chern, S.S., and Moser, J.K., Real hypersurfaces in complex manifolds, Acta Math. 133 (1974), 219-271; also Selected Papers, vol. 3, Springer-Verlag, New York, 1989, pp. 209-262.

[18] Cox, D., Little, J., and O'Shea, D., Ideals, Varieties, and Algorithms, 2nd ed., Springer-Verlag, New York, 1996.

[19] David, D., Kamran, N., Levi, D., and Winternitz, P., Subalgebras of loop algebras and symmetries of the Kadomtsev-Petviashivili equation, Phys. Rev. Lett. 55 (1985), 2111-2113.

[20] Di Francesco, P., Mathieu, P., and Sénéchal, D., Conformal Field Theory, Springer-Verlag, New York, 1997.

[21] Ehresmann, C., Introduction à la théorie des structures infinitésimales et des pseudo-groupes de Lie, in: Géometrie Différentielle, Colloq. Inter. du Centre Nat. de la Rech. Sci., Strasbourg, 1953, pp. 97-110.

[22] Evtushik, L.E., Pseudo-groups of transformations of geometrical-differential structures and their invariants, J. Math. Sci. 94 (1999), 1643-1684.

[23] Fefferman, C., and Graham, C.R., Conformal invariants, in: Élie Cartan et les Mathématiques d'aujourd'hui, Astérisque, hors série, Soc. Math. France, Paris, 1985, pp. 95-116.

[24] Fels, M., and Olver, P.J., Moving coframes. I. A practical algorithm, Acta Appl. Math. 51 (1998), 161-213.

[25] Fels, M., and Olver, P.J., Moving coframes. II. Regularization and theoretical foundations, Acta Appl. Math. 55 (1999), 127-208. 
[26] Fuchs, D.B., Gabrielov, A.M., and Gel'fand, I.M., The Gauss-Bonnet theorem and Atiyah-Patodi-Singer functionals for the characteristic classes of foliations, Topology 15 (1976), 165-188.

[27] Green, M.L., The moving frame, differential invariants and rigidity theorems for curves in homogeneous spaces, Duke Math. J. 45 (1978), 735-779.

[28] Griffiths, P.A., On Cartan's method of Lie groups and moving frames as applied to uniqueness and existence questions in differential geometry, Duke Math. J. 41 (1974), 775-814.

[29] Itskov, V., Orbit reduction of exterior differential systems and group-invariant variational problems, Contemp. Math. 285 (2001), 171-181.

[30] Itskov, V., Orbit Reduction of Exterior Differential Systems, Ph.D. Thesis, University of Minnesota, 2002.

[31] Janet, M., Leçons sur les Systèmes d'Équations aux Dérivées Partielles, Cahiers Scientifiques, fasc. IV, Gauthier-Villars, Paris, 1929.

[32] Jensen, G.R., Higher Order Contact of Submanifolds of Homogeneous Spaces, Lecture Notes in Math., vol. 610, Springer-Verlag, New York, 1977.

[33] Johnson, H.H., Classical differential invariants and applications to partial differential equations, Math. Ann. 148 (1962), 308-329.

[34] Kamran, N., Contributions to the study of the equivalence problem of Elie Cartan and its applications to partial and ordinary differential equations, Mém. Cl. Sci. Acad. Roy. Belg. 45 (1989), Fac. 7.

[35] Kamran, N., and Robart, T., A manifold structure for analytic isotropy Lie pseudogroups of infinite type, J. Lie Theory 11 (2001), 57-80.

[36] Kobayashi, S., Transformation Groups in Differential Geometry, Springer-Verlag, New York, 1972.

[37] Kogan, I.A., and Olver, P.J., The invariant variational bicomplex, Contemp. Math. 285 (2001), 131-144.

[38] Kogan, I.A., and Olver, P.J., Invariant Euler-Lagrange equations and the invariant variational bicomplex, Acta Appl. Math. 76 (2003), 137-193.

[39] Kumpera, A., Invariants différentiels d'un pseudogroupe de Lie, J. Diff. Geom. 10 (1975), 289-416.

[40] Kumpera, A., and Spencer, D., Lie Equations, Princeton University Press, Princeton, N.J., 1972.

[41] Kuranishi, M., On the local theory of continuous infinite pseudo groups I, Nagoya Math. J. 15 (1959), 225-260.

[42] Kuranishi, M., On the local theory of continuous infinite pseudo groups II, Nagoya Math. J. 19 (1961), 55-91.

[43] Lie, S., Über unendlichen kontinuierliche Gruppen, Christ. Forh. Aar. 8 (1883), 1-47; also Gesammelte Abhandlungen, Vol. 5, B.G. Teubner, Leipzig, 1924, pp. 314-360.

[44] Lie, S., Die Grundlagen für die Theorie der unendlichen kontinuierlichen Transformationsgruppen, Leipzig. Ber. 43 (1891), 316-393; also Gesammelte Abhandlungen, Vol. 6, B.G. Teubner, Leipzig, 1927, pp. 300-364. 
[45] Lisle, I.G., and Reid, G.J., Geometry and structure of Lie pseudogroups from infinitesimal defining systems, J. Symb. Comp. 26 (1998), 355-379.

[46] Lisle, I.G., and Reid, G.J., Cartan structure of infinite Lie pseudogroups, in: Geometric Approaches to Differential Equations, P.J. Vassiliou and I.G. Lisle, eds., Austral. Math. Soc. Lect. Ser., 15, Cambridge Univ. Press, Cambridge, 2000, pp. 116-145.

[47] Mackenzie, K., Lie Groupoids and Lie Algebroids in Differential Geometry, London Math. Soc. Lecture Notes, vol. 124, Cambridge University Press, Cambridge, 1987.

[48] Mansfield, E.L., Algorithms for symmetric differential systems, Found. Comput. Math. 1 (2001), 335-383.

[49] Marí Beffa, G., and Olver, P.J., Differential invariants for parametrized projective surfaces, Commun. Anal. Geom. 7 (1999), 807-839.

[50] McLachlan, R.I., and Quispel, G.R.W., What kinds of dynamics are there? Lie pseudogroups, dynamical systems and geometric integration, Nonlinearity 14 (2001), 1689-1705.

[51] Medolaghi, P., Classificazione delle equazioni alle derivate parziali del secondo ordine, che ammettono un gruppo infinito di trasformazioni puntuali, Ann. Mat. Pura Appl. 1 (3) (1898), 229-263.

[52] Morozov, O., Moving coframes and symmetries of differential equations, J. Phys. A 35 (2002), 2965-2977.

[53] Olver, P.J., Symmetry groups and group invariant solutions of partial differential equations, J. Diff. Geom. 14 (1979), 497-542.

[54] Olver, P.J., Applications of Lie Groups to Differential Equations, Second Edition, Graduate Texts in Mathematics, vol. 107, Springer-Verlag, New York, 1993.

[55] Olver, P.J., Equivalence, Invariants, and Symmetry, Cambridge University Press, Cambridge, 1995.

[56] Olver, P.J., Classical Invariant Theory, London Math. Soc. Student Texts, vol. 44, Cambridge University Press, Cambridge, 1999.

[57] Olver, P.J., Joint invariant signatures, Found. Comput. Math. 1 (2001), 3-67.

[58] Olver, P.J., Geometric foundations of numerical algorithms and symmetry, Appl. Alg. Engin. Commun. Comput. 11 (2001), 417-436.

[59] Olver, P.J., The canonical contact form, Adv. Studies Pure Math. 37 (2002), 267-285.

[60] Olver, P.J., and Pohjanpelto, J., Moving frames for Lie pseudo-groups, preprint, University of Minnesota, 2005.

[61] Olver, P.J., and Pohjanpelto, J., On the algebra of differential invariants of a Lie pseudo-group, preprint, University of Minnesota, 2005.

[62] Pommaret, J.F., Systems of Partial Differential Equations and Lie Pseudogroups, Gordon and Breach, New York, 1978.

[63] Pommaret, J.F., Partial Differential Equations and Group Theory, Kluwer Acad. Publ., Dordrecht, Netherlands, 1994. 
[64] Reid, G.J., Wittkopf, A.D., and Boulton, A., Reduction of systems of nonlinear partial differential equations to simplified involutive forms, Euro. J. Appl. Math. 7 (1996), 635-666.

[65] Riordan, J., An Introduction to Combinatorial Analysis, Princeton University Press, Princeton, N.J., 1980.

[66] Riquier, C., Les Systèmes d'Équations aux Dérivées Partielles, Gauthier-Villars, Paris, 1910.

[67] Robart, T., and Kamran, N., Sur la théorie locale des pseudogroupes de transformations continus infinis I, Math. Ann. 308 (1997), 593-613.

[68] Rodrigues, A.A.M., The first and second fundamental theorems of Lie for Lie pseudogroups, Amer. J. Math. 84 (1962), 265-282.

[69] Salmon, R., Lectures on Geophysical Fluid Dynamics, Oxford Univ. Press, Oxford, 1998.

[70] Singer, I.M., and Sternberg, S., The infinite groups of Lie and Cartan. Part I (the transitive groups), J. Analyse Math. 15 (1965), 1-114.

[71] Sternberg, S., Lectures on Differential Geometry, Prentice-Hall, Inc., Englewood Cliffs, N.J., 1964.

[72] Stormark, O., Lie's Structural Approach to PDE Systems, Cambridge University Press, Cambridge, 2000.

[73] Tsujishita, T., On variational bicomplexes associated to differential equations, Osaka J. Math. 19 (1982), 311-363.

[74] Vessiot, E., Sur l'intégration des systèmes différentiels qui admettent des groupes continues de transformations, Acta. Math. 28 (1904), 307-349.

[75] Vinogradov, A.M., and Krasil'shchik, I.S. (eds.), Symmetries and Conservation Laws for Differential Equations of Mathematical Physics, American Mathematical Society, Providence, RI, 1998.

[76] Weil, A., Oeuvres Scientifiques, vol. 1, Springer-Verlag, New York, 1979. 